\title{
Allergische Rhinitis im Kontext der chronischen Rhinosinusitis
}

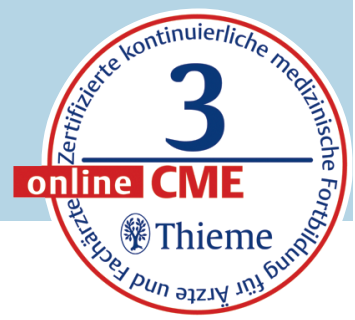

\section{Zusammenfassung \\ $\nabla$}

Die chronische Rhinosinusitis gehört zu den häufigsten chronischen Erkrankungen. Eine wichtige Ursache ist die allergische Rhinitis. Beide Erkrankungen und die Kombination von beiden, die im Mittelpunkt dieses Weiterbildungsbeitrags stehen, als auch die Abklärung und Behandlung der Differenzialdiagnosen gehören in die Hände des HNO-Arztes. Letztendlich ist nicht geklärt, warum eine allergische Rhinitis zu einer chronischen Rhinosinusitis führt. Zur Diagnostik und Therapie der allergischen Rhinosinusitis steht als Behandlungsrichtlinie eine Reihe von gut ausgearbeiteten nationalen und internationalen Leitlinien zur Verfügung. Dieser Beitrag stellt die wichtigsten aktuellen diagnostischen und therapeutischen Verfahren zur Behandlung der allergischen Rhinitis im Kindesalter und im Erwachsenenalter vor.

\section{Abstract \\ $\nabla$}

Chronic Rhinosinusitis is one of the most frequent chronic diseases. A major cause is allergic rhinitis. Both illnesses and the combination of both, which is the topic of this educational article, as well as the clarification and therapy of differential diagnoses are belonging in the hands of the otorhinolaryngologist. Finally, it is not completely understood which mechanisms of the allergic inflammation in the nose lead or predispose in the individual patient to the development of a chronic sinus disease. A series of excellent national and international guidelines have been published supporting the otorhinolaryngologist in diagnostics and treatment of patients with allergic rhinosinusitis. This article presents the most important and actual diagnostic and therapeutic procedures for the treatment of this important chronic disease in children and adults.

\section{Infobox}

Dieser Beitrag aus der Serie „Facharztwissen HNO“ in der Laryngo-Rhino-Otologie widmet sich dem Thema „Allergische Rhinitis im Kontext der chronischen Rhinosinusitis“. Über das Jahr verteilt erscheinen in unserer Rubrik „Facharztwissen HNO“ alle 2 Monate didaktisch aufbereitete Übersichtsartikel. Diese greifen die Hauptthemen der Kursreihe „Facharztwissen HNO“ auf, die im Wechsel an den HNO-Kliniken in Dresden, Erfurt, Jena, Leipzig, Magdeburg und Halle stattfindet (jährliche Veranstaltung der Professoren Andreas Dietz, Orlando GuntinasLichius, Thomas Zahnert, Dirk Eßer, Christoph

Arens und Stefan Plontke). Diese Kursreihe zielt erstrangig auf Assistenten in der Weiterbildung HNO-Heilkunde ab und soll in möglichst persönlichem Rahmen eine direkte Diskussion der Lehrinhalte im Rahmen eines interaktiven Workshops ermöglichen. Die Autoren haben sich zur Aufgabe gemacht, die Inhalte praxisgerecht mit Rücksicht auf aktuelle wissenschaftliche Entwicklungen anzubieten. Der Inhalt des HNOFacharztwissens unterliegt einem ständigen Fluss, sodass durch das Konsensusverfahren der 6 genannten ausgewiesenen Fachvertreter die Aktualität und Belastbarkeit des angebotenen Wissens angestrebt wird.
Videos zu diesem Beitrag finden Sie im Internet bei Ihrer LRO an unter: http://dx.doi.org/

$10.1055 / \mathrm{s}-0035-1547290$

\section{Video 1}
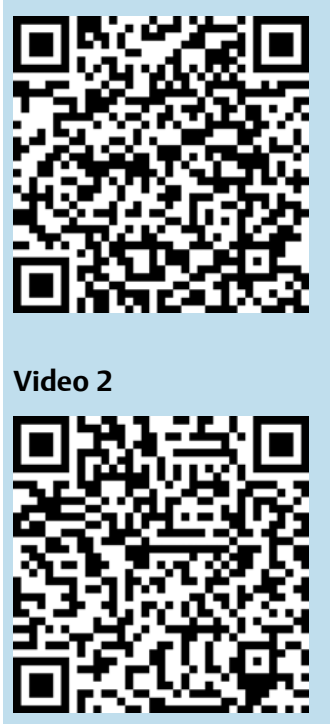


\section{Einleitung}

Zur Rhinosinusitis (RS) gehören die akute Rhinosinusitis (ARS) und die chronische Rhinosinusitis (CRS). Die ARS und die CRS gehören zu den häufigsten Erkrankungen des Menschen überhaupt. Ist die RS mit Allergien assoziiert, so spricht man von einer allergischen Rhinitis. Die allergische Rhinitis ist für sich genommen erst einmal eine Erkrankung der Nase und ist im akuten Stadium nicht durch eine klinisch symptomatische Sinusitis gekennzeichnet. Anderseits ist die Schleimhaut der Nasennebenhöhlen auch von der allergischen Erkrankung betroffen, sodass manche Autoren immer von einer Rhinosinusitis sprechen. Nichtsdestotrotz wird im vorliegenden Artikel gemäß dem internationalen Sprachgebrauch der Begriff der „allergischen Rhinitis“ gebraucht.

Merke: Eine chronische Sinusitis ist genau genommen eine mögliche Folge der allergischen Erkrankung.

Diese Übersichtsarbeit zum Facharztwissen orientiert sich an den nationalen und internationalen Leitlinien [1-5]. Die Diagnostik und Therapie der allergischen RS und der CRS werden dargestellt, wobei sich die Diagnostik und Therapie der allergischen RS und der CRS überschneiden können.

\section{Definition}

Eine RS ist im Positionspapier „EPOS 2012“ der European Rhinologic Society und der European Academy of Allergy and Clinical Immunology definiert als eine Entzündung der Schleimhaut der Nase und der Nasennebenhöhlen [6]. Da in der Regel die Schleimhäute von Nase und Nasennebenhöhlen gemeinsam von der Erkrankung betroffen sind, wird von einer „Rhinosinusitis“ und nicht getrennt von einer „Rhinitis“ und „Sinusitis“ gesprochen. Eine ARS hat eine Dauer von weniger als 12 Wochen. Ab einer Dauer von 12 Wochen spricht man von einer CRS. Hat ein Patient 4 Episoden einer ARS pro Jahr, also zwischen den Episoden immer ein symptomfreies Intervall, so spricht man von einer rezidivierenden ARS. Ist der Patient dazwischen nicht symptomfrei, so lautet die Diagnose korrekt CRS mit rezidivierender Exazerbation (Infobox). Bei der CRS unterscheidet man dann noch die CRS mit Nasenpolypen von der CRS ohne Nasenpolypen. Unter Nasenpolypen versteht man eine chronisch-entzündliche Erkrankung der sinunasalen Schleimhaut, die zu dem typischen klinischen Bild der ödematösen Schleimhauthyperplasie führt (๑ Abb. 1, Video 1).

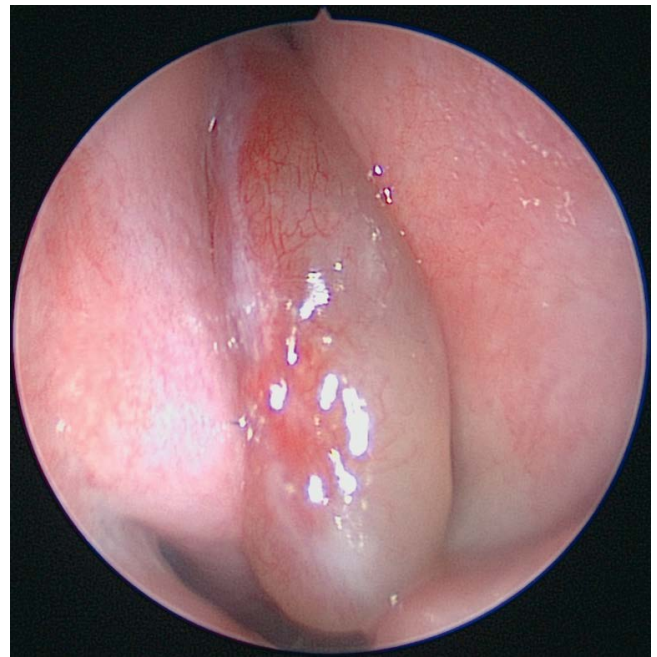

Abb. 1 Patient mit Vorgeschichte einer allergischen Rhinitis bei Birkenpollenallergie seit über 10 Jahren. Nun Vorstellung mit Symptomatik einer nasalen Obstruktion außerhalb der Pollensaison. Die Nasenendoskopie zeigt beidseitig Nasenpolypen im mittleren Nasengang, hier die rechte Seite gezeigt. Das polypöse Gewebe drängt aus dem mittleren Nasengang zwischen untere Nasenmuschel (links im Bild) und Nasenscheidewand (rechts im Bild).

\section{Video 1}

Nasenendoskopie eines Patienten mit allergischer Rhinitis und Nasenpolypen im mittleren Nasengang beidseitig

\section{Definitionen der Rhinosinusitis}

- akute Rhinosinusitis (ARS) < 12 Wochen Dauer

- rezidivierende ARS=4 Episoden einer ARS pro Jahr

- chronische Rhinosinusitis (CRS) $\geq 12$ Wochen Dauer

Die allergische Rhinitis passt nicht richtig in dieses Definitionsschema. Grundsätzlich liegt hierbei eine allergische Entzündung der Nasen- und Nasennebenhöhlenschleimhaut vor auf Basis einer IgE-vermittelten Sofortreaktion des Immunsystems auf körperfremde Stoffe. Da die Entwicklung einer klinischen Symptomatik durch eine Allergie im strengen Sinne nicht akut geschieht, sprechen die WHO-Richtlinien Allergic Rhinitis and its Impact on Asthma (ARIA) von einer intermittierenden allergischen Rhinitis bei einer Dauer von $<4$ Tagen/Woche oder $<28$ Tagen insgesamt. Dagegen spricht man von einer persistierenden allergischen Rhinitis bei einer Dauer von $>4$ Tagen/Woche oder $>28$ Tagen insgesamt [1]. Hierbei stellen die Begriffe „intermittierend“ und „persistierend“ die Dauer der Symptomatik in den Vordergrund und haben die alte Klassifikation mit den Begriffen „saisonal“ und „perennial“ abgelöst, da eine saisonale Allergie, z.B. durch Gräser auch viele Monate andauern könnte 
oder anderseits eine perenniale Allergie, z.B. durch Milben, saisonalen Schwankungen unterliegen kann. In der Literatur findet sich auch noch der Begriff der „allergischen Rhinokonjunktivitis“. Hiermit wird hervorgehoben, dass die Patienten nicht nur Beschwerden mit der Nase sondern auch mit den Augen wie z.B. durch Bindehautentzündungen oder ein Lidödem haben können. Manchmal wird der Begriff auch mit der Laienbezeichnung „Heuschnupfen“ gleichgesetzt. Der Begriff „Atopie“ beschreibt die persönliche oder familiäre Prädisposition, sollte der Betroffene einem bestimmten Allergen exponiert werden, eine Typ-I-Immunreaktion zu entwickeln. Zu den atopischen Erkrankungen zählt neben der allergischen RS auch das Asthma bronchiale und das atopische Ekzem.

\section{Definition allergische Rhinitis}

- intermittierende allergische Rhinitis $<4$ Tagen/Woche Dauer oder $<28$ Tagen insgesamt

- persistierende allergische Rhinitis $\geq 4$ Tagen/ Woche Dauer oder $>28$ Tagen insgesamt

\section{Allergische Reaktionsformen}

$\nabla$

Coombs und Gell haben die Antwort des Immunsystems auf Antigene in 4 Klassen aufgeteilt. Obwohl es sich dabei um eine starke Vereinfachung handelt, wird diese Einteilung auch heute noch verwendet:

- Typ I beschreibt die IgE-vermittelte Reaktion. Wenn ein Allergen an ein spezifisches zellgebundenes IgE-Molekül bindet, die sich z.B. auch auf Mastzellen und basophilen Granulozyten befinden, dann degranulieren diese Zellen und setzen Mediatoren wie Histamin und Leukotriene frei. Das sind Botenstoffe, die die allergische Entzündungsreaktion hervorrufen. Die ersten Symptome einer Typ-IReaktion treten schon nach wenigen Minuten auf (Frühphase). Davon ist die Spätphase abzugrenzen, die durch Zytokine von T-Lymphozyten und eosinophilen Granulozyten vermittelt wird. Die in diesem Artikel abgehandelte allergische Rhinosinusitis ist eine typische Folge einer Typ-I-Reaktion. Weitere wichtige Formen sind:

- allergischer Schock

- allergisches Asthma bronchiale

- Insektengiftallergie

- verschiedene Formen von Nahrungsmittelallergien

- Typ II ist eine zytotoxische Reaktion, bei der sich das Antigen direkt auf einer Zellmembran befindet; diese Reaktion tritt z. B. bei den Blutgruppenantigenen auf. Durch die Reaktion der zellgebundenen Antigene mit zirkulierenden IgG- oder IgM-Antikörpern wird eine Lyse der Zelle ausgelöst.

- Typ III beschreibt eine Komplexbildung zwischen zirkulierenden Antigenen und spezifischen Antikörpern (überwiegend IgG-Antikörper).

- Typ IV schließlich ist die verzögerte Immunreaktion nach 24-48 Stunden, ausgelöst durch T-Zellen, die mit spezifischen T-Zell-Rezeptoren Antigene erkennen.

- Tab. 1 stellt zusammenfassend die Unterschiede der 4 Klassen mit klinischen Beispielen dar.

Merke: Die allergische Rhinitis ist eine allergische Entzündung der Nasen- und Nasennebenhöhlenschleimhaut auf Basis einer IgE-vermittelten Sofortreaktion des Immunsystems auf körperfremde Stoffe (Typ-I-Reaktion nach Coombs und Gell).

\section{Epidemiologie \\ $\nabla$}

Die allergische RS tritt mit einer Prävalenz von 15-25\% der Gesamtbevölkerung in Deutschland auf. Die Prävalenz bei Kleinkindern liegt bei $1-15 \%$ und bei Schulkindern bei $2-40 \%$, wobei Jungen häufiger betroffen sind als Mädchen. Nachdem die Sensibilisierungsraten über die letzten 15 Jahre angestiegen sind, scheint nun in Deutschland ein Plateau erreicht worden zu sein. Ist kein Elternteil Atopiker, so liegt das Atopierisiko bei $10-15 \%$; ist ein Elternteil Atopiker bei $30-40 \%$ und wenn beide Eltern Atopiker sind bei $50-80 \%$. Etwa $10-30 \%$ aller chronischen Sinusitiden sind allergiebedingt. Mehr als 40\% der Patienten mit

\begin{tabular}{|c|c|c|c|}
\hline Tyр & $\begin{array}{l}\text { Intervall zwischen Exposi- } \\
\text { tion mit dem Antigen und } \\
\text { der Immunreaktion }\end{array}$ & Mechanismus & Typische allergische Erkrankungen \\
\hline 1 & sofort & $\begin{array}{l}\text { IgE-vermittelte Freisetzung von } \\
\text { Mediatoren }\end{array}$ & $\begin{array}{l}\text { - allergische Rhinosinusitis } \\
\text { - anaphylaktischer Schock } \\
\text { - Asthma bronchiale }\end{array}$ \\
\hline II & Minuten & $\begin{array}{l}\text { zytotoxische lgG- und IgM- } \\
\text { Antikörper }\end{array}$ & - Transfusions-Zwischenfall \\
\hline III & 3-8 Stunden & zirkulierende Immunkomplexe & $\begin{array}{l}\text { - Lupus erythematodes } \\
\text { - Purpura Schönlein-Henoch }\end{array}$ \\
\hline IV & 24-72 Stunden & zytotoxische T-Zell-Reaktion & $\begin{array}{l}\text { - Kontaktekzem } \\
\text { - Arzneimittelexanthem }\end{array}$ \\
\hline
\end{tabular}


intermittierender allergischer Rhinitis entwickeln binnen 10 Jahren ein allergisches Asthma bronchiale. Umgekehrt weisen 30-50\% der Asthmatiker eine intermittierende allergische Rhinitis und etwa $20 \%$ eine persistierende allergische Rhinitis auf. Patienten mit CRS und Asthma bronchiale weisen zu 30\% eine Analgetika-Intoleranz auf. Umgekehrt haben Patienten mit AnalgetikaIntoleranz und Asthma in $60 \%$ eine CRS, und zwar mit Nasenpolypen. Unter allen Formen der CRS ist die allergische Rhinitis die häufigste Ursache: Etwa $60 \%$ aller Patienten mit CRS (mit oder ohne Nasenpolypen) weisen eine Typ-I-Allergie gegen Inhalationsallergene auf.

Merke: Die allergische Rhinitis hat eine hohe Prävalenz von 15-25\% in der deutschen Bevölkerung. Etwa $10-30 \%$ aller chronischen Sinusitiden sind allergiebedingt.

\section{Ätiologie}

$\nabla$

Die Entwicklung einer allergischen Erkrankung resultiert aus einem multikausalen Geschehen aus genetischer Prädisposition und Umweltfaktoren. Die genetische Komponente der allergischen Rhinitis ist polygen wahrscheinlich mit einem komplexen Vererbungsmuster. In Bezug auf die Umweltfaktoren ist der Einfluss des aktiven und passiven Rauchens belegt.

Merke: Kinder rauchender Eltern habe ein erhöhtes Risiko eine atopische Erkrankung zu entwickeln.

Luftschadstoffe scheinen auch zu einer allergischen Sensibilisierung beizutragen. Eine verminderte frühkindliche Exposition gegenüber mikrobiellen Stimuli scheint ebenfalls die Sensibilisierung zu erhöhen (Hygienehypothese), wobei hierzu teilweise widersprüchliche Untersuchungsergebnisse vorliegen. Der Zusammenhang zwischen der allergischen Entzündung der Nase und der Entwicklung einer chronischen Sinusitis ist bislang nicht ausreichend geklärt. Dies ist auch aus therapeutischem Blickwinkel bedeutsam (siehe auch Kapitel „Therapie“). Behandlungskonzepte, die evidenzbasiert hocheffektiv bei allergischer Rhinitis sind, haben sich teilweise bei der CRS als weniger oder gar nicht effektiv erwiesen [7].

Merke: Zwar finden sich unter den Patienten mit CRS viele mit einer allergischen Rhinitis, doch ist der pathogenetische Zusammenhang nicht geklärt.

\section{Wichtigste Allergene}

$\nabla$

Die Epidemiologie der wichtigsten Allergene variiert standortbedingt sehr, daher sollen hier nur exemplarisch wenige wichtige Allergene aufgeführt werden. Eine gute Datenquelle zur Suche und Information über allergene Moleküle stellt z.B. die Allergom-Datenbank dar (http://www. allergome.org/).

In der Zeit von Februar bis September sind typische Inhalationsallergene verantwortlich für eine intermittierende allergische Rhinitis. Dies sind die Pollen von:

- Erle

$\checkmark$ Hasel

- Birke

- Gräser

- Roggen

- Beifuß

- Wegerich

Die Pollen stammen von windbestäubenden Pflanzen mit großen Pollenmengen. Insektenbestäubte Pflanzen spielen wegen der wesentlich geringeren Pollenmengen eine untergeordnete Rolle. Pilzsporen können auch zu einer intermittierenden allergischen RS führen.

Typische Allergene, die eine persistierende allergische RS auslösen, sind:

- Hausstaubmilbe

- Tierallergene

- Schimmelpilze

- Nahrungsmittel

- Kontaktallergene

Die Immunantwort ist immer gegen bestimmte Proteine des allergenen Agens gerichtet. Und von den relevanten Proteinen wird in der Regel nur ein Teil als bestimmte Konformation oder Sequenz als Epitop erkannt. Das eigentliche Allergen ist also kein Pollentyp, wie z.B. Birkenpollen-1, sondern ein Bestandteil des Pollens (bei Birkenpollen-1 z.B. Bet v1). Dieses eigentliche Allergen kann anderswo in der Natur auftreten, was Kreuzreaktivitäten erklärt. So kommt z.B. Bet v1 auch homolog in Haselpollen, Äpfeln oder Pfirsichen vor, was wiederum erklärt, warum eine aerogene Sensibilisierung auch zu einer (zusätzlichen) Nahrungsmittelallergie führen kann.

\section{Diagnostik}

$\nabla$

Um die Diagnose einer allergischen Rhinitis und gleichzeitig einer CRS stellen zu können, müssen der Nachweis einer allergischen Erkrankung und die Kriterien für die Diagnose einer CRS gleichzeitig geführt werden. Die wichtigsten Untersuchungsverfahren sind:

- Anamnese

- HNO-Untersuchung einschließlich Nasenendoskopie

- Bildgebung mit Computertomografie

- Allergietestung 


\begin{tabular}{|c|c|c|c|}
\hline Merkmal & Kontrolliert & $\begin{array}{l}\text { Teilweise kontrolliert } \\
\text { (1-2 Merkmale positiv) }\end{array}$ & $\begin{array}{l}\text { Unkontrolliert ( } \geq 3 \text { Merkmale } \\
\text { der Kategorie teilweise } \\
\text { kontrolliert positiv) }\end{array}$ \\
\hline nasale Obstruktion & nicht vorhanden/störend & $\begin{array}{l}\text { an den meisten Tagen der } \\
\text { Woche }\end{array}$ & $\begin{array}{l}\text { an den meisten Tagen der } \\
\text { Woche }\end{array}$ \\
\hline Rhinorrhoe & nein oder nur mukoserös & mukopurulent & mukopurulent \\
\hline Gesichtsschmerz & nicht vorhanden/störend & ja & ja \\
\hline Riechstörung & $\begin{array}{l}\text { nicht oder nur geringfü- } \\
\text { gig vorhanden }\end{array}$ & ja & ja \\
\hline Schlafstörung & nicht vorhanden/störend & ja & ja \\
\hline $\begin{array}{l}\text { Nasenendoskopie- } \\
\text { befund }\end{array}$ & unauffällig & $\begin{array}{l}\text { pathologisch (Nasenpolypen, } \\
\text { entzündete Schleimhaut) }\end{array}$ & $\begin{array}{l}\text { pathologisch (Nasenpolypen, } \\
\text { entzündete Schleimhaut) }\end{array}$ \\
\hline $\begin{array}{l}\text { systemische Medi- } \\
\text { kation erforderlich }\end{array}$ & nein & $\begin{array}{l}\text { Antibiotika oder Kortikosteroi- } \\
\text { de in den letzten } 3 \text { Monaten }\end{array}$ & $\begin{array}{l}\text { Antibiotika oder Kortikosteroi- } \\
\text { de in letzten Monaten }\end{array}$ \\
\hline
\end{tabular}

Bei der Allergietestung nehmen diagnostische Tests, die in vivo und in vitro den Nachweis von freien oder zellgebundenen IgEs führen, eine zentrale Rolle ein. Nach vollständiger Diagnostik soll der HNO-Arzt in der Lage sein, den Schweregrad der Erkrankung einzuteilen anhand der 3 Kategorien:

- leichtgradig

- mittelgradig

- schwer

Zusätzlich ist für die Therapieplanung die Einschätzung der Symptomkontrolle wichtig. Auch hierfür werden 3 Kategorien empfohlen:

- kontrolliert

- teilweise kontrolliert

- unkontrolliert

Ist über $\geq 1$ Jahr die Erkrankung trotz bestmöglicher Therapie unkontrolliert, so spricht man von einer therapierefraktären CRS (Difficult-to-treatCRS). Anamnese und Untersuchung erlauben anhand des in 0 Tab. 2 abgebildeten Schemas eine Einteilung der CRS anhand der Symptomkontrolle. $\odot$ Abb. 2 gibt einen Überblick über die notwendige Diagnostik zur Diagnose allergische RS, $\odot$ Abb. $\mathbf{3}$ und $\odot$ Abb. 4 eine erste Übersicht über Diagnostik und Therapie der CRS in Abhängigkeit vom Vorhandensein von Nasenpolypen. Der allergische Aspekt der Erkrankung wird in diesen beiden Abbildungen nicht detailliert betrachtet.

Merke: Wird die Diagnose einer CRS gestellt, sollte dazu auch der Schweregrad (leichtgradig, mittelgradig, schwer) und der aktuelle Zustand der Symptomkontrolle (kontrolliert, teilweise kontrolliert, unkontrolliert) genannt werden.

\section{Anamnese}

Die Anamnese geht auf den Symptomenkomplex der allergischen Komponente der Erkrankung und der CRS ein. Die Symptome überschneiden sich (s. Infobox). Die Kardinalsymptome der allergischen Komponente der Erkrankung sind:

- Niesen

- Juckreiz

- klare Sekretion

- nasale Obstruktion

Die pollenbedingte Rhinitis zeichnet sich vor allem durch Niesen, Sekretion und Begleitkonjunktivitis aus, während z.B. milbenbedingte Rhinitiden als wichtigstes Symptom eine Obstruktion verursachen. Sekundäre Symptome der allergischen Reaktion können sein:

- Husten

- Halsschmerzen

- Pharyngitis

- Konjunktivitis

- Lidödeme

- Laryngitis

Eine hilfreiche Fragen-Checkliste zur Diagnose einer allergischen Rhinitis zeigt $\bigcirc$ Tab. 3. Nach bekannten Allergien wird genauso gefragt wie nach relevanten Komorbiditäten in Form von anderen atopischen Erkrankungen (Asthma, Ekzem). Eine Analgetika-Intoleranz wird hinterfragt. Die nasale Hyperreaktivität ist ein weiteres wichtiges Merkmal der allergischen Komponente: Die Patienten zeigen häufig eine verstärkte Reaktion auf unspezifische Reize (Tabakrauch, Stäube, Geruchsstoffe, Temperaturänderungen) in Form von Niesen, nasaler Obstruktion und/ oder Sekretion.

Merke: Es kann sinnvoll sein, die nasale Hyperreaktivität durch eine Provokation mit kalter Luft zu objektivieren [8].

Die Leitsymptome der CRS werden abgefragt:

- nasale Obstruktion

- anteriore und/oder posteriore Rhinorrhoe

- Mittelgesichtsdruck/Schmerz

- Hyposmie

- Schlafstörung 
Mindestens 2 dieser Symptome werden für die Diagnosestellung gefordert. Die Frage nach der Dauer der Beschwerden ist wichtig, da Beschwerden 12 Wochen oder mehr das Kriterium für die chronische RS ist.

\section{Kardinalsymptome}

Allergische Rhinitis:
- Niesen
- Juckreiz der Nase
- klare Sekretion
- nasale Obstruktion
Chronische Rhinosinusitis:
- nasale Obstruktion
- anteriore und/oder posteriore Rhinorrhoe
- Mittelgesichtsdruck/Schmerz
- Hyposmie
- Schlafstörung

\section{HNO-ärztliche und allgemeine körperliche Untersuchung}

Zusätzlich wird für die Diagnose der CRS der Nachweis eines pathologischen Befunds gefordert; das kann entweder in Form eines durch beidseitige Nasenendoskopie erhobenen pathologischen endonasalen Befunds und/oder durch einen pathologischen CT-Befund geschehen. Eine anteriore Rhinoskopie nur mit dem Nasenspekulum wird von vielen im Rahmen der Diagnostik für nicht ausreichend erachtet.

Merke: Die Nasenendoskopie ist wichtig für die Trennung der CRS in CRS mit Nasenpolypen und CRS ohne Nasenpolypen.

Typische endoskopische Befunde der allergischen Erkrankung sind:

- Schwellung der unteren Muschel

- teilweise auch Schwellung der mittleren Muschel

- schleimig-wässrige Sekretion

Die Schleimhaut kann ödematös und bläulich livide verfärbt erscheinen. Befunde wie Septumdeviation oder eine Nasenmuschelhyperplasie werden ebenso dokumentiert. Bei der Untersuchung ist auch die Betrachtung der äußeren Nase, der Augen und umgebenden Haut wichtig; ekzematöse Veränderungen der Haut um den Naseneingang weisen auf eine Hypersekretion und/ oder allergische Reaktion hin. Überhaupt wird auf Atopiezeichen geachtet.

Für die Bewertung der Schwere der Erkrankung wird empfohlen, den Patienten eine $10 \mathrm{~cm}$ visuelle Analogskala (VAS), skaliert von 0-10, vorzulegen. Leichtgradige Beschwerden entsprechen

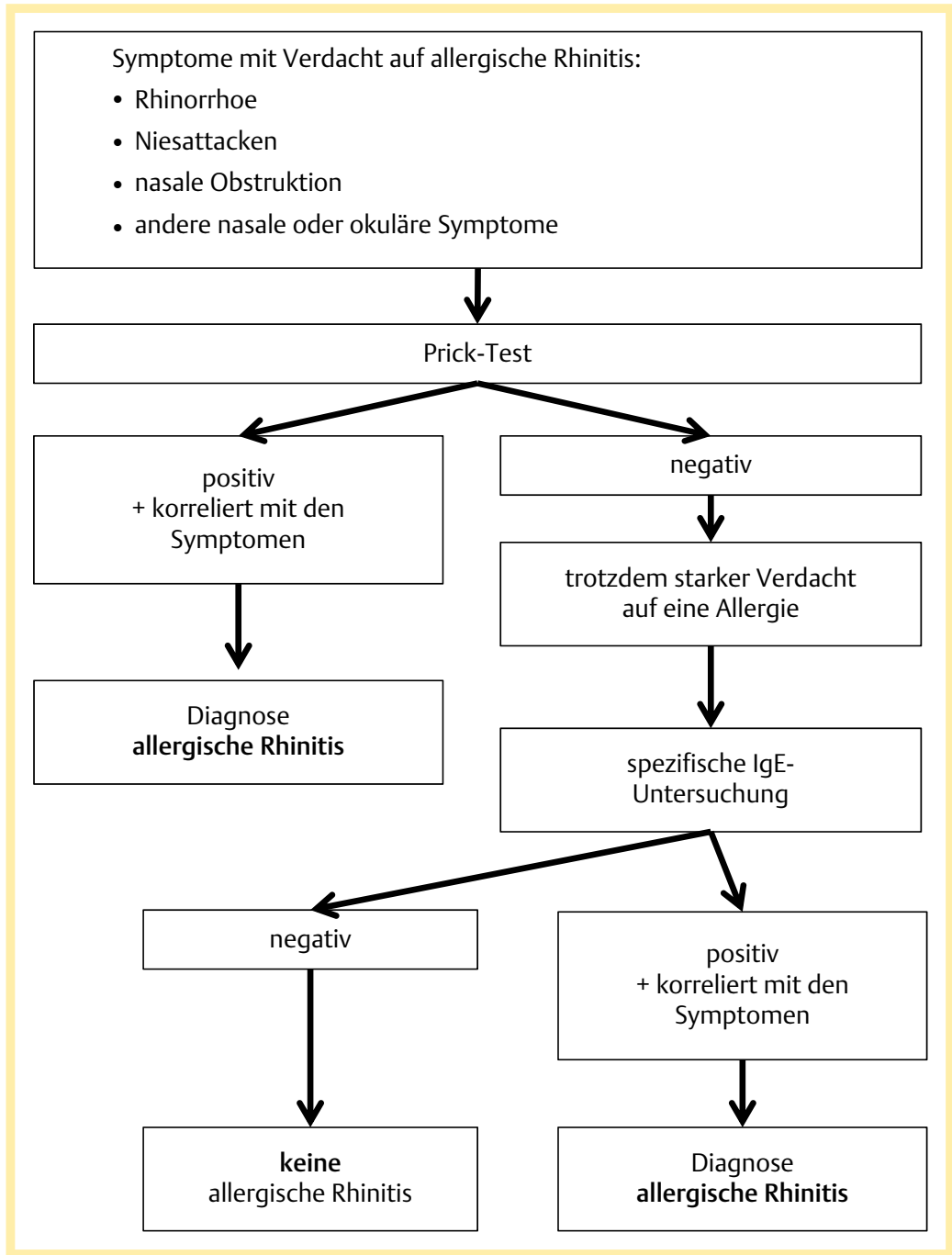

Abb. 2 Algorithmus zur Diagnostik bei Verdacht auf allergische Rhinitis (modifiziert nach [42]).

dabei 0-3 cm, mittelgradige Beschwerden 3-7 cm und schwere Beschwerden 7-10 cm auf der Skala. Insbesondere bei Patienten mit mittelgradiger bis schwerer Symptomatik und/oder persistierender Symptomatik sollte auch eine Abklärung eines Asthma bronchiale, ggf. unter Hinzuziehung anderer Fachkollegen, erfolgen.

Merke: Wird die Diagnose einer allergischen Rhinitis gestellt, so sollte insbesondere bei mittelgradiger bis schwerer Symptomatik und/oder persistierender Symptomatik auch eine Abklärung eines Asthma bronchiale erfolgen.

\section{Hauttestung}

$\nabla$

Cave: Die Hauttestung selbst kann, wenn auch selten, zu einer anaphylaktischen Reaktion führen. 
Abb. 3 Algorithmus für das Management der chronischen Rhinosinusitis ohne Nasenpolypen (CRSsNP) bei Erwachsenen durch HNO-Fachärzte. Im violett hinterlegten Kasten rechts oben sind besondere differenzialdiagnostische Umstände sowie Hinweise auf drohende Komplikationen aufgeführt. Die im mittleren Kasten aufgeführte Langzeitantibiose (gemeint sind Makrolide in niedriger Dosierung) hat einen niedrigen Evidenzlevel (nach [6]).

Abb. 4 Algorithmus für das Management der chronischen Rhinosinusitis mit Nasenpolypen (CRSwNP) bei Erwachsenen durch HNO-Fachärzte. Die im mittleren Kasten aufgeführte Anwendung von Tropfen statt Spray beruht auf Untersuchungen, dass Tropfen bei Applikation in Kopfhängelage besser wirken als Sprays. Die ebenfalls aufgeführte Tetrazyklin-Behandlung hat einen niedrigen Evidenzlevel (nach [6]).
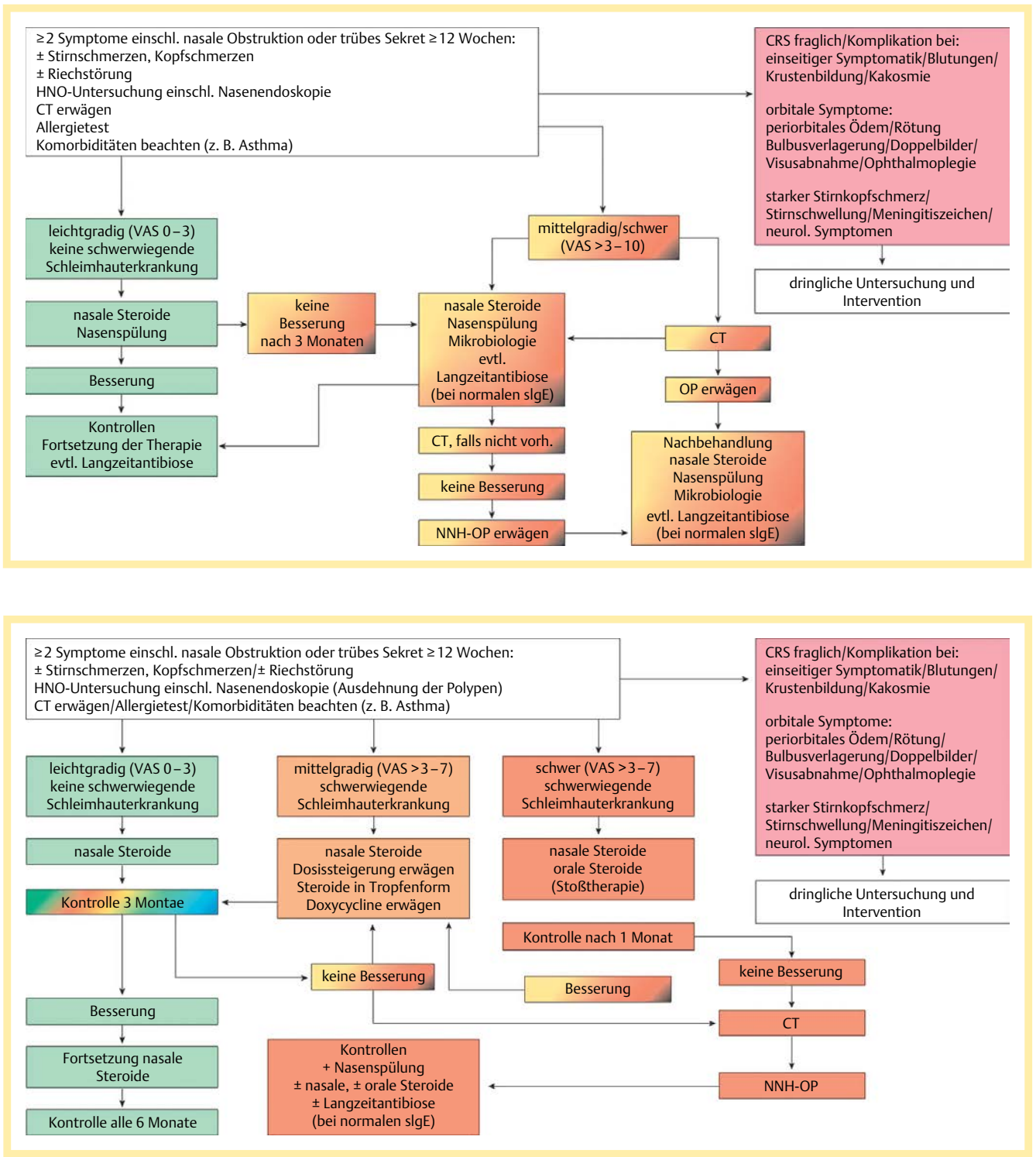

Darüber muss der Patient aufgeklärt sein und entsprechende Vorsicht geübt werden. Vor einer Hauttestung ist zudem zu beachten, dass Medikamente eine potenzielle allergische Reaktion unterdrücken können. Insbesondere orale Kortikosteroide in höherer Dosierung sollten für eine Woche oder Antihistaminika mind. 3 Tage vor der Testung nicht eingenommen werden.

Merke: Wenn die Positivkontrolle mit Histamin negativ ausfällt, ist immer auszuschließen, ob nicht andere Medikamente die Reaktion unterdrückt haben.

Der Pricktest mit standardisierten Allergenextrakten (seltener mit experimentellen Extrakten oder rekombinanten Allergenen) an der Volarseite des Unterarms ist der wichtigste diagnostische Hauttest zum Nachweis IgE-vermittelter allergischer Sofortreaktionen ( $\bullet$ Abb. 5; Infobox) [9]. Hierbei wird das Allergen mithilfe einer Lanzette in die obere Dermis eingebracht, ohne dass es zu einer Blutung kommen sollte. Bei sensibilisierten Patienten sind allergenspezifische IgE-Antikörper auf der Oberfläche von dermalen Mastzellen gebunden. Bei Antigenbindung wird eine Kaskade von Mastzellreaktionen mit Freisetzung vasoaktiver Mediatoren in Gang gesetzt. So kommt es zu der typischen umschriebenen Quaddelbildung an der Prick-Stelle. Ein negativer Prick-Test kann auch zur differenzialdiagnostischen Abgrenzung nicht IgE-vermittelter, klinisch jedoch ähnlicher, Reaktionen dienen, wie der Intoleranzreaktion gegen nicht steroidale Antiphlogistika. Kontraindikationen für einen Prick-Test sind:

- Hauterkrankungen im Testgebiet

- nicht kontrolliertes Asthma bronchiale

- stark reduzierter Allgemeinzustand

Hatte der Patient unlängst eine allergische Soforttyp-Reaktion, sollte der Prick-Test erst nach einer Refraktärperiode von 4 Wochen erfolgen. 
Merke: Ein positiver Prick-Test hat nur dann eine Bedeutung für die Diagnosestellung einer allergischen Rhinitis, wenn auch eine passende klinische Symptomatik vorliegt. Im Zweifel sind weitere Allergietestungen notwendig.

\section{Grundsätze der Prick-Testung}

- Karenzzeit für Medikamente mit Einfluss auf den Prick-Test beachten

- Refraktärperiode nach einer SoforttypReaktion vor dem Prick-Test beachten

- Testung an der Volarseite des Unterarms oder auf dem Rücken (bei Hautveränderungen an den Unterarmen)

- Allergenspektrum anhand der Anamnese festlegen

- Negativkontrolle mit physiologischer Kochsalzlösung und Positivkontrolle mit Histaminhydrochlorid-Lösung

- Ablesen nach $15 \mathrm{~min}$

- Test positiv=mittlerer Quaddeldurchmesser $\geq 3 \mathrm{~mm}$, wenn Negativkontrolle negativ
Auch der Intrakutantest (in der Regel auf dem Rücken) dient dem Nachweis einer Soforttyp-Reaktion. Er ist sensitiver aber unspezifischer als der Prick-Test. Der Test ist aufgrund der intradermalen Injektion schmerzhafter als der Prick-Test und wird daher bei Kindern selten angewandt. Liegt anamnestisch der hochgradige Verdacht auf eine allergische Sensibilisierung vor, bleibt aber der Prick-Test und die Labordiagnostik negativ, kann im Einzelfall der Intrakutantest indiziert sein.

Der Epikutantest und der Atopie-Patchtest sind zur Abklärung einer T-Zell-vermittelten Spättyp-Reaktion interessant, also vor allem bei Kontaktallergenen. Diese sind im Zusammenhang mit der allergischen Rhinosinusitis nicht von direktem Interesse. Die praktische Durchführung der Hauttests ist ausführlich z. B. in der entsprechenden Leitlinie der Deutschen Gesellschaft Allergologie und klinische Immunologie beschrieben (AWMF Nr. 061/026).

Merke: Die wichtigsten Hauttests zur Abklärung einer allergischen Rhinitis sind der Prick-Test (erste Wahl) und der Intrakutantest.

Tab. 3 Fragen-Checkliste zur allergischen Rhinitis (modifiziert nach: [42]).

\begin{tabular}{|c|c|c|}
\hline Frage & Antwort & \\
\hline \multicolumn{3}{|l|}{ Frage 1: Haben Sie die folgenden Beschwerden? } \\
\hline Beschwerden nur auf einer Nasenseite? & ja & nein \\
\hline Dicker, grüner oder gelber Auswurf aus der Nase? ${ }^{*}$ & ja & nein \\
\hline Läuft eingedicktes Sekret von der Nase in den Rachen? Oder haben Sie eine laufende Nase?* & ja & nein \\
\hline Gesichtsschmerz?* & ja & nein \\
\hline Wiederholtes Nasenbluten? & ja & nein \\
\hline Riechverlust? $^{*}$ & ja & nein \\
\hline \multicolumn{3}{|c|}{$\begin{array}{l}\text { Frage 2: Haben Sie die folgenden Beschwerden zumindest } 1 \text { Stunde an den meisten Tagen (oder an den meisten Tagen } \\
\text { zu einer bestimmten Jahreszeit)? }\end{array}$} \\
\hline Wässriges Naselaufen? & ja & nein \\
\hline Niesattacken? & ja & nein \\
\hline Nase verstopft? & ja & nein \\
\hline Nasenjucken? & ja & nein \\
\hline Augenrötung, Augenjucken? & ja & nein \\
\hline
\end{tabular}

* typische Symptome einer Sinusitis

Frage 1: Symptome aus Frage 1 sind nicht typisch für eine allergische Rhinitis

Frage 2: wässrige Rhinorrhoe und mind. 1 weiteres Symptom aus Frage 2
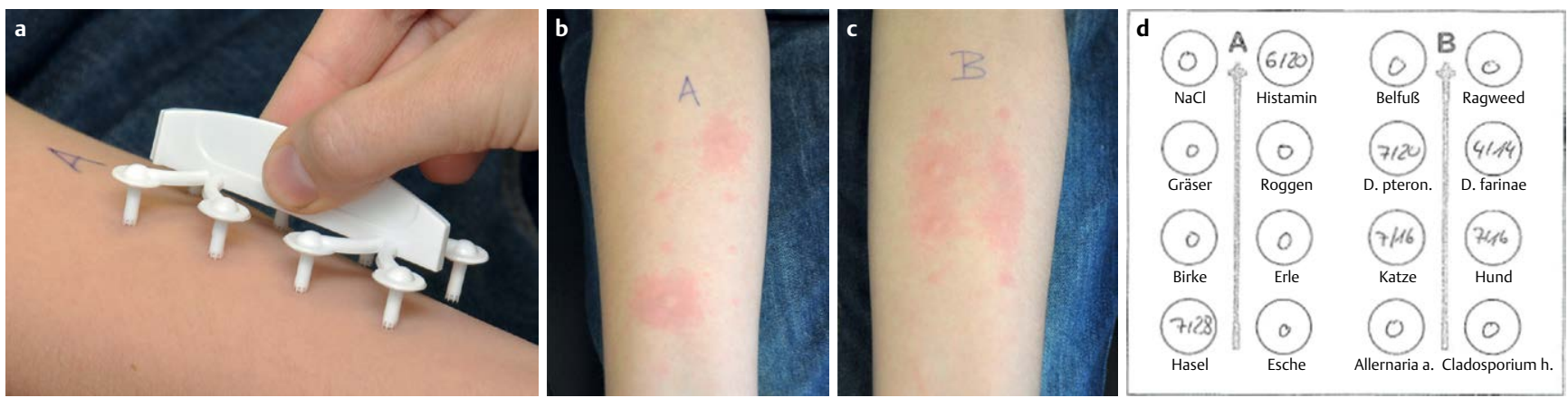

Abb. 5 Prick-Test. a Pricken der Haut mit einem Prick-System, das die gleichzeitige Applikation von 8 Prick-Lösungen erlaubt. b Reaktion des rechten Arms. c Reaktion des linken Arms. d Dokumentation der Ergebnisse des rechten und linken Arms: Histamin-Probe positiv. Starke Reaktion auf Hasel, Dermatophagoides pteronyssinus und Dermatophagoides farinae, Katze und Hund passend zur Anamnese des Patienten. Erste Zahl: Quaddeldurchmesser; Zweite Zahl: Hautrötung in mm. 


\section{In-vitro-Diagnostik \\ $\nabla$}

Liegt eine Diskrepanz zwischen den Befunden der Hauttestungen und der allergologischen Anamnese vor, so kann die serologische Testung sinnvoll sein. Ist bei einem Patienten eine hochgradige Anaphylaxie bekannt mit erhöhtem Risiko einer erneuten schweren Reaktion im Rahmen der Prick- oder Intrakutantestung, so ist ebenso die In-vitro-Diagnostik wichtig. Die wichtigsten Untersuchungen sind die Bestimmung des Gesamt-IgE und von spezifischen IgE-Antikörpern. Die Höhe der spezifischen IgE-Antikörper ist altersabhängig und immer im Kontext des Gesamt-IgE zu bewerten. Es ist wichtig daran zu erinnern, dass ein erhöhter IgE-Serumspiegel nicht nur die Summe allergenspezifischer IgE-Antikörper bildet, sondern dass dieser auch auf einer genetischen Prädisposition beruhen oder Ausdruck nicht atopischer Grunderkrankungen sein kann. Der Nachweis eines spezifischen IgE-Antikörpers ist nicht gleichbedeutend mit einer klinischen Relevanz, sondern bedeutet nur, dass bei dem Patienten eine spezifische Sensibilisierung vorhanden ist. Der Serumspiegel des Gesamt-IgE und allergenspezifischer IgEAntikörper wird in vitro in internationalen Units (IU/ml) quantifiziert. Zelluläre Testverfahren (Basophilen-Aktivierungstest, Lymphozytentransformationstest usw.) spielen in der Routinediagnostik der allergischen Rhinitis keine Rolle.

Merke: Der Nachweis eines spezifischen lgEAntikörpers bedeutet für sich allein genommen nur, dass der Patient eine spezifische Sensibilisierung aufweist. Zu einer Krankheit wird der Befund nur im Kontext mit Symptomatik und den Ergebnissen der Haut-Testung.

\section{Nasaler Provokationstest \\ $\nabla$}

Der nasale Provokationstest, in der Regel mit kommerziellen Allergenextrakten, ist zur Bestätigung der klinischen Relevanz einer Sensibilisierung geeignet. Der Test hat eine hohe Sensibilität und Spezifität. Er kann auch hilfreich sein, wenn anamnestisch kein klarer Zusammenhang zwischen Exposition, Sensibilisierung und Symptomatik zu erkennen ist. Wenn die Patienten nach Hauttestung und/oder Labordiagnostik mehrere Allergene aufweisen, kann die nasale Provokation helfen, die klinisch relevanten Allergene zu erkennen. Wie bei allen anderen Provokationsformen ist zu beachten, ob der Patient Medikamente einnimmt, die Provokationsreaktionen erschweren können ( $\beta$-Blocker, ACE-Hemmer). Eine Kontraindikation ist wiederum ein unkontrolliertes Asthma bronchiale. Hat der Patient eine Nasen- oder Nasennebenhöhlenoperation erhalten, so sollte ein Abstand von mind. 2 Monaten beachtet werden. In der Regel sind die Aller- genextrakte als isotonische und $\mathrm{pH}$-neutrale Allergenextrakte (also anders aufbereitet als beim Prick-Test) kommerziell erhältlich. Als Negativkontrolle wird die Trägersubstanz verwendet. Mehr Details finden sich in der Infobox und $\bullet$ Tab. 4.

Merke: Der nasale Provokationstest kann hilfreich sein, wenn die sonstige Befundkonstellation unklar oder widersprüchlich und der Patient nach Hauttestung und/oder Labordiagnostik mehrere Allergene aufweist und die klinisch relevanten Allergene erkannt werden sollen.

Tab. 4 Symptomscore des nasalen Provokationstests.

\begin{tabular}{|l|l|l|}
\hline \multirow{3}{*}{ Symptomatik } & Stufen & Punkte \\
\hline \multirow{2}{*}{ Irritation } & $0-2 x$ Niesen & 0 \\
\cline { 2 - 3 } Sekretion & $3-5 x$ Niesen & 1 \\
\cline { 2 - 3 } & $>5 x$ Niesen & 2 \\
\cline { 2 - 3 } Fernsymptome & kein Sekret & 0 \\
\cline { 2 - 3 } & wenig Sekret & 1 \\
\cline { 2 - 3 } & viel Sekret & 2 \\
\cline { 2 - 3 } & $\begin{array}{l}\text { keine } \\
\text { Tränenfluss und/oder }\end{array}$ & 0 \\
\hline & $\begin{array}{l}\text { Gaumenjucken und/oder } \\
\text { Ohrenjucken }\end{array}$ & 1 \\
\cline { 2 - 3 } & $\begin{array}{l}\text { Konjunktivitis und/oder Che- } \\
\text { mosis und/oder Urtikaria und/ } \\
\text { oder Husten und/oder Luftnot }\end{array}$ & 2 \\
\hline
\end{tabular}

Grundsätze der nasalen Provokationstestung

- Karenzzeit für Medikamente mit Einfluss auf die nasale Provokation beachten

- Abstand von mind. 2 Monaten nach einer Nasenoperation beachten

- Raumtemperatur $20 \pm 1,5^{\circ} \mathrm{C}$

- Akklimatisation des Patienten über 15-30 min

- Messung auf der Nasenseite mit der besseren Atmung

- 1-2 Sprühstöße auf untere/mittlere Nasenmuschel, während der Patient nach tiefem Luftholen die Luft anhält

- Erheben des initialen Symptomscores (० Tab. 4)

- erste Messung ohne Provokation (Ausgangswert)

- zweite Messung mit Negativkontrolle (Leerwert) nach 10 min Wartezeit: Symptomscore $+R$ hinomanometrie

Flow-Reduktion $>20 \%$ bei 150 Pa: Testabbruch Flow-Reduktion $<20 \%$ : Fortsetzung

- dritte Messung mit Allergenlösung nach 10 min Wartezeit: Symptomscore+Rhinomanometrie Flow-Reduktion $>40 \%$ /Symptomscore $>3$ / Test positiv Flow-Reduktion $>20 \%+$ Symptomscore $>2$ | Test positiv bei negativem Test Wiederholung der Messung nach $10 \mathrm{~min}$

- Testung weiterer Allergene 


\section{Bildgebung}

Bildgebung der Wahl zur Diagnosestellung einer CRS ist eine Computertomografie (CT) der Nasennebenhöhlen $[3,10]$. Der Endoskopiebefund korreliert deutlich besser mit dem CT-Befund als die beklagten Symptome:

- Bei einer CRS ohne Nasenpolypen hilft die CT die Ausdehnung der Entzündung zu bestimmen.

- Bei einer CRS mit Nasenpolypen hilft die CT die Ausdehnung der Nasenpolypen zu bestimmen.

In beiden Fällen ist eine CT besonders wichtig, wenn der Patient nicht auf eine medikamentöse Therapie anspricht. Ist der Patient bereits voroperiert, so dient die CT dazu, die Ausdehnung der Voroperation im Hinblick auf eine erneute Operation zu erfahren und mögliche Gefahrenstellen zu erkennen. Generell gilt heute eine CT der Nasennebenhöhlen zur Operationsplanung als obligatorisch. Zum Nutzen der digitalen Volumentomografie (DVT) bei CRS liegen keine ausreichenden Publikationen vor.

Merke: Eine CT der Nasennebenhöhlen ist wichtig bei Patienten, die nicht auf eine medikamentöse Therapie der CRS ansprechen und bei denen eine Nasennebenhöhlenoperation geplant ist.

\section{Differenzialdiagnostik}

Abhängig davon, wie die allergische Rhinitis verläuft, müssen andere Formen der ARS oder CRS differenzialdiagnostisch ausgeschlossen werden. Zudem kann es sein, dass beim Patienten allergische Beschwerden bekannt sind, aber eine andere Ursache zum Beschwerdebild oder veränderten Beschwerdebild beiträgt.

Seltene Ursachen einer CRS sind eine ziliäre Dyskinesie oder eine zystische Fibrose. Entsprechend tritt die Erkrankung im frühen Lebensalter auf. Die CRS kann Manifestationsort einer systemischen Erkrankung wie dem M. Wegener, dem Churg-Strauss-Syndrom oder einer Sarkoidose sein. Bei einseitiger Manifestation mit Betonung auf der Erkrankung in der Kieferhöhle muss eine dentogene Ursache ausgeschlossen werden. Unklar ist die Bedeutung anatomischer Varianten, die zu Engstellen in der Nasennebenhöhlendrainage oder einer Septumdeviation führen. Es gibt sowohl Untersuchungen, die eine Assoziation zeigen als auch solche, die keinen Zusammenhang erkennen können. Eine pilzbedingte CRS scheint in Deutschland selten zu sein. Der Einfluss von Umweltschadstoffen als auch von klimatischen Bedingungen ist letztendlich nicht eindeutig geklärt.

Bei einem akuten Verlauf muss differenzialdiagnostisch an eine die akute virale RS, die akute postvirale RS und an die akute bakterielle RS gedacht werden. Am häufigsten ist davon die akute virale RS. Aus dieser entsteht bei etwa $10 \%$ der Patienten die postvirale Form. Dabei halten die Symptome mehr als 10 Tage an, oder werden um den 5. Krankheitstag deutlich schlimmer. Auch die bakterielle RS basiert häufig auf einer zunächst viralen RS und macht nur $1 \%$ der Fälle aus. Eine wichtige Differenzialdiagnose ist die Analgetika-Intoleranz (ASS-Intoleranz, Morbus Widal, Samter-Trias). Die Patienten weisen keine Allergien auf, beklagen aber im Vollbild einen Symptomkomplex aus nasalen Beschwerden (Obstruktion, Rhinorrhoe, Hyposmie), Polyposis nasi und nicht allergischem Asthma bronchiale. Die Beschwerden werden durch die Einnahme von nicht steroidalen Antiphlogistika ausgelöst. Zusätzlich haben 2-37\% der Patienten gastrointestinale Entzündungsreaktionen bis Ulzerationen, 5-15\% Angioödeme und 20-45\% eine Urtikaria.

Schließlich gilt es bei Patienten, bei denen eine CRS bereits bekannt ist, auszuschließen, dass eine akute Exazerbation durch eine bakterielle Superinfektion für die Beschwerden verantwortlich ist. Hierbei kann es sich um eine typische Superinfektion einer viralen Entzündung oder direkte bakterielle Entzündung handeln. Dabei ist zu beachten, dass das Erregerspektrum bei der CRS, insbesondere bei voroperierten Patienten, anders ist als bei der ARS, z.B. Staphylokokken eine wesentlich größere Rolle spielen [11]. Die Abgrenzung einer Differenzialdiagnose von einer komorbiden Erkrankung kann manchmal schwierig sein, da Patienten mit CRS per se eine erhöhte Prävalenz für komorbide Erkrankungen aufweisen (z.B. anderen Atemwegsinfektionen oder auch eine Refluxerkrankung) [12].

\section{Prävention \\ $\nabla$}

Oberste Priorität haben die Verminderung der Ursachen der Allergieentstehung (Primärprävention), insbesondere bei Risikogruppen mit genetischer Vorbelastung, und die Verhinderungen einer manifesten Erkrankung bei am besten symptomlosen Personen mit frühen Krankheitszeichen oder zur Vermeidung eines Symptomwechsels (Sekundärprävention).

Daher stehen Kinder im Fokus der Prävention. Stillen über die ersten 4 Lebensmonate ist bspw. eine effektive präventive Maßnahme [2]. Aktive und passive Exposition gegenüber Tabakrauch erhöht das Allergierisiko (als auch das Sinusitisrisiko) $[13,14]$. Dagegen scheint die Adipositas im Kindesalter kein Risikofaktor für eine allergische RS oder eine CRS zu sein [15]. Eine ausführliche Zusammenfassung über die Evidenzlage zur Prävention von Allergien liegt mit der S3-Leitlinie Allergieprävention (Nr. 061/016) vor. 


\section{Therapie}

$\nabla$

Die Therapieentscheidung hängt vor allem von:

- Schwere der Symptomatik

- Allergenspektrum

- Patientenwunsch

Die Faustregeln der Therapie sind in 0 Abb. 6 dargestellt und lauten wie folgt: Da eine allergische Erkrankung der Nase vorliegt, erfolgt primär eine Behandlung der allergischen Komponente der Erkrankung. Therapie der ersten Wahl ist eine medikamentöse Therapie. Ist diese nicht erfolgreich oder ausreichend, sollte eine spezifische Immuntherapie erfolgen. Eine Chirurgie an den unteren Nasenmuschel oder eine Septumplastik kann indiziert sein, wenn die nasale Obstruktion als Hauptsymptom trotz adäquater Pharmakotherapie persistiert. Eine antientzündliche Medikation in Kombination mit Solespülungen ist der erste Schritt der Behandlung der CRS. Diese Therapie wird dann schrittweise der erfolgten Symptomkontrolle angepasst. Ist eine medikamentöse Langzeittherapie erfolglos, wird die Indikation zur Nasennebenhöhlenchirurgie geprüft. Es gibt zusätzlich ein großes Angebot an komplementärmedizinischen Verfahren zur Diagnostik und Therapie allergischer Erkrankungen, so auch der allergischen RS. Da für kein komplementärmedizinisches Verfahren eine Wirksamkeit nachgewiesen ist, wird aus Platzgründen hier auf eine Darstellung verzichtet. Die wichtigsten Therapieziele sind:

- ungestörter Schlaf

- normales Alltagsleben ohne Einschränkungen

- keine belastenden Symptome

- allenfalls geringe Nebenwirkungen durch die Behandlung

\section{Karenz}

$\nabla$

Theoretisch ist die Karenz des auslösenden Allergens die beste Behandlung. Wenn mehrere Allergene relevant sind, besteht häufig nicht die Möglichkeit der Karenz. Eine Reduktion der Expositionsmenge allein ist aber meist bereits sinnvoll, da dies die Beschwerden lindern kann. Anders als beim allergischen Asthma verringert aber z.B. bei der Haustaubmilbenallergie ein Encasing zur Verringerung der Hausstaubmilbenlast nicht die Symptome der allergischen Rhinitis [16].

\section{Medikamentöse Therapie \\ $\nabla$}

Hier gibt es Überschneidungen in den Behandlungsempfehlungen zur allergischen Rhinitis und zur Behandlung der CRS. Die Grundzüge der Behandlung der CRS sind bereits in $\triangle$ Abb. 3 und $\odot$ Abb. 4 und die der allergischen Rhinitis in $\odot$ Abb. 6 dargestellt worden. Da bis dato nicht genau geklärt ist, wie eine allergische Erkran- kung der Nase zu einer CRS führt, ist die Rolle der antiallergischen Komponente der Therapie der RS bei Patienten mit klinisch relevanter allergischer Rhinitis und CRS unklar (ausführlich diskutiert in: [1]). Deswegen wird die medikamentöse Therapie der allergischen Rhinitis und der CRS in $\odot$ Tab. 5 getrennt dargestellt. Nicht aufgenommen wurden in diese tabellarische Darstellungen Therapieverfahren, die sich evidenzbasiert als unwirksam erwiesen haben (z.B. topische oder systemische Antimykotika). Eine adjuvante mehrwöchige Therapie durch Nasenspülungen mit isotoner Kochsalzlösung kann die Symptome der allergischen Komponente als auch der CRS lindern [17]. Eine Reihe von älteren Arbeiten konnte zeigen, dass die Einnahme von Bakterienlysaten die Beschwerden lindern können [18]. Aktuelle Arbeiten zum Nutzen liegen hierzu nicht vor, doch finden sich nichtsdestotrotz Bakterienlysate auch in den aktuellen Behandlungsempfehlungen [4]. Leiden die Patienten auch unter Augensymptomen der allergischen Rhinitis, so sind orale H1-Antihistaminika, topische H1-Antihistaminika und topische Cromone zu empfehlen. Bei Patienten mit CRS können Episoden/Exazerbationen von akuten Sinusitiden durch eine bakterielle Superinfektion auftreten. Eine orale Antibiotikatherapie $<4$ Wochen ist hilfreich, wohingegen Langzeittherapien ( $>12$ Wochen) allenfalls nur einen geringen Effekt haben $[4,19]$.

\section{Spezifische Immuntherapie}

Die spezifische Immuntherapie (SIT) in Form der subkutanen Immuntherapie (SCIT) und der sublingualen Immuntherapie (SLIT) sind nachweislich effektive Behandlungen, um die Symptome der allergischen Rhinitis dauerhaft zu verbessern und den Bedarf an Medikamenten zu reduzieren [20,21].

Trotzdem wird vermutet, dass im deutschsprachigen Raum weiterhin eine Unterversorgung der Patienten mit dieser Therapieoption besteht [22]. Die SIT ist die einzige Behandlungsmethode, die zu einer Toleranz gegenüber dem relevanten Allergen führen kann.

Merke: Die subkutane Immuntherapie (SCIT) und die sublinguale Immuntherapie (SLIT) sind derzeit die wichtigsten, weil effektivsten Formen der spezifischen Immuntherapie (SIT).

Die SCIT ist die älteste und am besten untersuchte Form der SIT; insbesondere bei einer konsequenten Behandlungsdauer über 3 Jahre. Die SCIT ist nachweislich bereits im ersten Jahr nach Therapiebeginn häufig effektiver als eine medikamentöse Therapie [23]. Doch auch zur SLIT zur Behandlung mit Gräserpollenextrakten gibt es mittlerweile Metaanalysen zur Wirksamkeit bei 


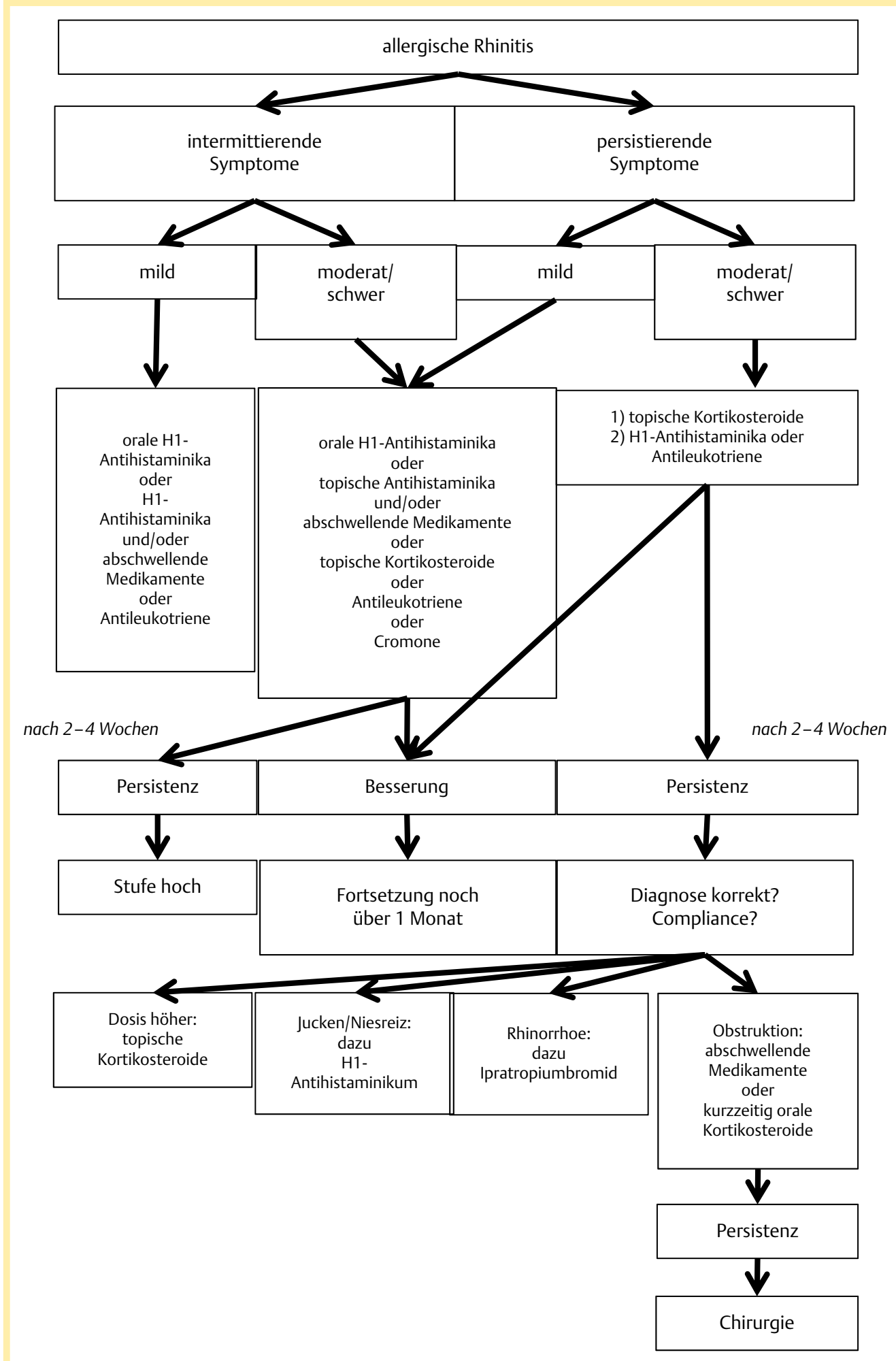

Abb. 6 Stufenalgorithmus für die medikamentöse Behandlung der allergischen Rhinitis anhand des Schweregrads (modifiziert nach [42]).
Erwachsenen und Kindern [24]. Für die SLIT gibt es derzeit in Deutschland wesentlich weniger zugelassene Allergenextrakte als für die SCIT. Daher ist - zumindest derzeit - ein Vergleich von SLIT und SCIT nicht sinnvoll. Indikationen und Kontraindikation der SIT sind in $\odot$ Tab. 6 aufgelistet.

Merke: Eine Sensibilisierung gegen mehrere Allergene ist keine Kontraindikation für die SIT.
Die Auswahl der Allergenextrakte orientiert sich an der Symptomatik: in einem frühen Erkrankungsstadium und bei Erreichen der Peak-Dosis ist die SIT effektiver. Eine gute Wirksamkeit ist ab dem Alter von 5 Jahren belegt. Durch die guten Behandlungserfolge bereits nach 30 Tagen mit einer SLIT mit Gräserpollen [25], wird mehr und mehr die Notwendigkeit einer jahrelangen SIT hinterfragt. Bspw. wurde die Cluster-SCIT einge- 
Tab. 5 Häufige Therapieformen und deren evidenzbasierter Empfehlungsgrad.

\begin{tabular}{|c|c|c|c|c|c|c|c|}
\hline \multirow{3}{*}{ Therapie } & \multicolumn{4}{|c|}{ Allergische Rhinitis } & \multicolumn{3}{|c|}{ Chronische Rhinosinusitis } \\
\hline & \multicolumn{2}{|c|}{ intermittierend } & \multicolumn{2}{|l|}{ persistierend } & \multirow{2}{*}{$\begin{array}{l}\begin{array}{l}\text { ohne Nasen- } \\
\text { polypen }\end{array} \\
\text { Erwachsene }\end{array}$} & \multirow{2}{*}{$\begin{array}{l}\text { mit Nasen- } \\
\text { polypen } \\
\text { Erwachsene }\end{array}$} & \multirow[b]{2}{*}{ Kinder } \\
\hline & Erwachsene & Kinder & Erwachsene & Kinder & & & \\
\hline orale H1-Antihistaminika & A & A & A & A & $\mathrm{D}$ & $\mathrm{D}$ & - \\
\hline $\begin{array}{l}\text { topische H1-Antihista- } \\
\text { minika }\end{array}$ & A & A & A & A & - & - & - \\
\hline topische Kortikosteroide & A & A & A & A & A & A & $\mathrm{D}$ \\
\hline orale Kortikosteroide & - & - & - & - & C & A & - \\
\hline topische Cromone & A & A & A & A & - & - & - \\
\hline Anti-Leukotriene & A & $\begin{array}{l}\mathrm{A}(>6 \\
\text { Jahre) }\end{array}$ & A & - & - & $\mathrm{A} !^{*}$ & - \\
\hline topische Dekongestiva & - & - & - & - & D & D & \\
\hline Solespülung & - & - & - & - & A & D & A \\
\hline Bakterienlysate & - & - & - & - & A & - & - \\
\hline Mukolytika & - & - & - & - & C & $\mathrm{D}$ & \\
\hline Antirefluxtherapie & - & - & - & - & $\mathrm{D}$ & C & C \\
\hline Allergenkarenz & $\mathrm{D}$ & D & A & B & $\mathrm{D}$ & - & - \\
\hline $\begin{array}{l}\text { subkutane spezifische } \\
\text { Immuntherapie }\end{array}$ & A & A & A & A & - & - & - \\
\hline $\begin{array}{l}\text { sublinguale spezifische } \\
\text { Immuntherapie }\end{array}$ & A & A & A & B & - & - & - \\
\hline
\end{tabular}

$A=$ direkt basierend auf Kategorie I-Evidenz

$\mathrm{B}=$ direkt basierend auf Kategorie II-Evidenz oder extrapoliert auf Kategorie I-Evidenz

$\mathrm{C}=$ direkt basierend auf Kategorie III-Evidenz oder extrapoliert auf Kategorie II-Evidenz

$\mathrm{D}=$ direkt basierend auf Kategorie IV-Evidenz oder extrapoliert auf Kategorie III-Evidenz

* Behandlung evidenzbasiert als unwirksam belegt

Tab. 6 Indikationen und Kontraindikationen der spezifischen Immuntherapie.

\begin{tabular}{l|l}
\hline Indikationen & Kontraindikation \\
\hline - Nachweis einer IgE-vermittelten Sensibili- & - Polysensibilisierung ist keine Kontraindikation! \\
sierung mit klinischer Symptomatik & - teil- oder unkontrolliertes Asthma bronchiale \\
- Symptome $\geq 2$ Jahre & - kardiovaskuläre Erkrankung mit erhöhtem Risiko bei Adrenalingabe \\
- Allergenkarenz nicht möglich & - Behandlung mit Beta-Blockern \\
- Therapie-Compliance über 2-3 Jahre mit & - synchrone maligne Erkrankung \\
dem Patienten konsentiert & - schwere Autoimmunerkrankung oder Erkrankung des Immunsystems
\end{tabular}

führt, bei der die Dosissteigerungen der SCIT beschleunigt werden, um die Gesamtbehandlungsdauer zu verringern. Aus evidenzbasierter Sicht muss festgestellt werden, dass die Datenlage zur Effektivität der Cluster-SCIT noch nicht ausreichend ist, um das Verfahren abschließend zu bewerten [26]. Aus diesem Grund wird in dieser Übersichtsarbeit auch nicht näher auf andere Dosierungsschemata (Kurzzeit-Immuntherapie, Rush, Ultra-Rush) eingegangen. Bis ausreichend Langzeitstudien vorliegen, bleibt es bei der Empfehlung, die SLIT oder SCIT über 3 Jahre zu planen.

Merke: Wie lange der Therapieerfolg einer SIT anhält und was wichtige Prädiktoren sind, ist bislang unzureichend untersucht.

Will man die Therapie individualisieren, so kann die Behandlung auch fortgesetzt werden, z.B. 1 Jahr über eine Beschwerdefreiheit hinaus oder bis keine weitere klinische Besserung mehr beobachtet werden kann. Die Sensibilisierung bleibt auch nach Abschluss der SIT im Hauttest oder bei den In-vitro-Tests nachweisbar und hat keinen Bezug zum Therapieerfolg.
Die Dauer des Therapieerfolgs ist unterschiedlich lang. Es liegen nur wenige gute Untersuchungen zur Dauer des Therapieeffekts, in der Regel über Jahre anhaltend, vor. Die Durchführung der SIT ist übersichtlich in einer S2-Leitlinie der Deutschen Gesellschaft Allergologie und klinische Immunologie beschrieben (AWMF Nr. 061/004).

\section{Chirurgische Therapie \\ $\nabla$}

Bei persistierender nasaler Obstruktion bei allergischer Rhinitis trotz leitliniengerechter medikamentöser Behandlung ist die chirurgische Verkleinerung der unteren Nasenmuscheln eine gute therapeutische Option. Es wird immer wieder bemängelt, dass es an kontrollierten klinischen Studien zur Effektivität der Muschelchirurgie mangelt [27]. Hinzu kommt, dass eine Vielzahl an technischen Verfahren zur Verkleinerung der Nasenmuscheln beschrieben ist. Die Chirurgie verfolgt das Ziel, die Querschnittsfläche der Nasenhöhlen im Bereich der Nasenklappe zu vergrößern, den nasalen Widerstand zu verringern und den Luftstrom durch die Nase zu vergrößern. Die transnasale endoskopische Nasennebenhöh- 


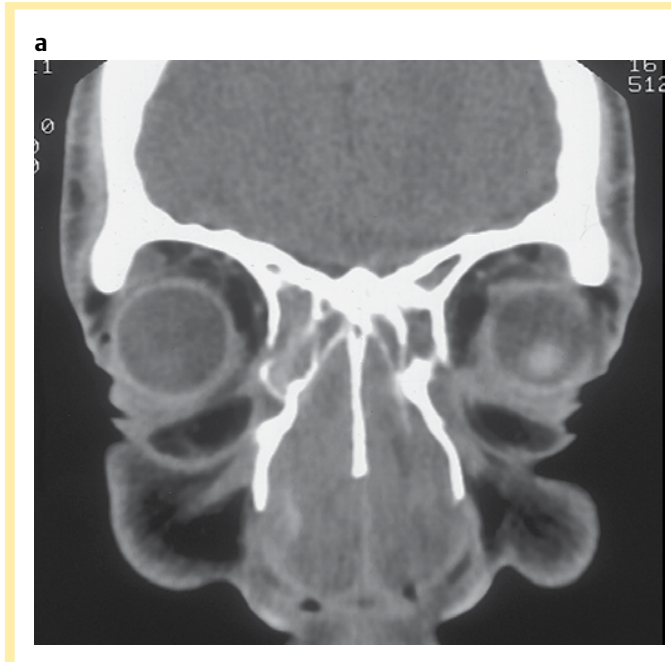

b

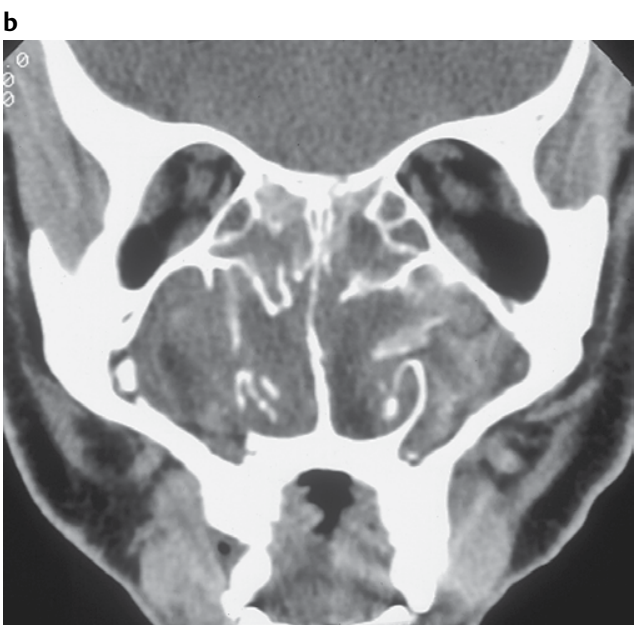

\section{Video}

Ausschnitt aus einer typischen funktionellen endoskopischen Nasennebenhöhlenchirurgie. Operation der linken Seite, Entfernung von Nasenpolypen aus der Nasenhaupthöhle. Das linke hintere Siebbein ist bereits teilweise von Polypen befreit.

lenchirurgie hat sich als effektive Behandlungsmaßnahme für Patienten mit CRS und persistierenden Symptomen trotz adäquater medikamentöser Therapie erwiesen ( $\bullet$ Abb. 7; $\odot$ Video 2 ) [28-30].

Merke: Die funktionelle endoskopische Nasennebenhöhlenchirurgie (functional endoscopic sinus surgery; FESS) ist anerkanntermaßen das wirksame chirurgische Verfahren zur Behandlung einer CRS, wenn eine medikamentöse Therapie nicht ausreicht.

\section{Therapierefraktäre CRS}

$\nabla$

Wenn ein Patient trotz adäquater medikamentöser oder chirurgischer Therapie weiter unter einer beeinträchtigten Symptomatik leidet, so spricht man von einer unkontrollierten (therapierefraktären) CRS [31]. Daher ist zu prüfen:

- Wurde die korrekte Therapieauswahl getroffen (wenn Alternativen bestehen)?

- Welcher Anwendungsweg (lokal, systemisch) wurde gewählt?

- Erfolgt die Therapie tatsächlich symptomorientiert?

- Nimmt der Patient die Medikamente korrekt ein?

- War die Diagnosestellung sowohl der allergischen Erkrankung als auch der CRS korrekt (siehe Differenzialdiagnostik)?

Konkomitante anatomische Pathologien wie eine Septumdeviation oder im Bereich der osteomeatalen Einheit, eine zusätzliche pulmonale oder
Systemerkrankung sind auszuschließen. Es kann auch sein, dass eine nasale Hyperreaktivität das führende Symptom ist. Bei der allergischen Komponente der Erkrankung kann auch der inflammatorische Teil der Erkrankung die führenden Symptome ausmachen, sodass dieser Teilaspekt therapeutisch intensiver angegangen werden muss. Bei Kindern muss an eine Nasenrachenblockade durch hyperplastische Adenoide gedacht werden. Ist der Patient mit CRS voroperiert, so muss ggf. durch einen Beta-Trace-Test ausgeschlossen werden, dass die persistierende Rhinorrhoe nicht durch ein Liquorleck durch einen Schädelbasisdefekt verursacht ist.

\section{Allergische Rhinosinusitis bei Kindern \\ $\nabla$}

Bei Kindern mit allergischer Rhinitis, die eine CRS aufweisen, besteht möglicherweise ein engerer Zusammenhang zwischen der allergischen Erkrankung und der chronischen Nasennebenhöhlenentzündung [32]. Das macht die grundlegende Behandlung der allergischen Erkrankung bedeutsam. Bei Kindern steht die Fremd- und Elternanamnese im Vordergrund. Zur allergischen RS bei Kindern gibt es wesentlich weniger Handlungsempfehlungen in den Leitlinien, hinzu kommt die Symptomüberlappung mit den Beschwerden bei Hyperplasie der Adenoide. Erfolgt die Diagnosestellung anhand der Kriterien in $\odot$ Tab. 2, so beobachtet man bei vielen Kindern eine Heilung allein durch eine Adenotomie! Dies erläutert eingängig das diagnostische Dilemma bei Kindern.

Zur Behandlung von Kindern gibt es im Vergleich zur Sachlage bei Erwachsenen nur wenige klinische Studien mit hohem Evidenzniveau. Daher erreicht der Empfehlungsgrad für bestimmte Therapieformen zumeist nicht das hohe Niveau wie bei den Therapieempfehlungen für Erwachsene. Es ist wichtig festzuhalten, dass nicht nur die spezifische Immuntherapie, die medikamentöse Therapie, sondern auch die chirurgische
Abb. 7 Koronare Computertomografie der Nasennebenhöhlen. Massive Polyposis der Nase und der Nasennebenhöhlen beidseitig trotz intensiver medikamentöser Therapie und wiederholter ambulanter Polypektomie vor geplanter FESS [43]. 
Therapie als funktionelle endoskopische Nasennebenhöhlenchirurgie eine wirksame und sichere Therapie bei Kindern mit CRS ist [33].

Merke: Wird bei Kindern mit CRS über chirurgische Maßnahmen nachgedacht, gehört die Adenotomie mit in das Therapiekonzept.

\section{Leitlinien}

$\nabla$

Im Onlineportal der Arbeitsgemeinschaft der Wissenschaftlichen Medizinischen Fachgesellschaften (AWMF; http://www.awmf.org/leitlinien.html) findet sich eine im Jahr 2014 überarbeitete S3-Leitlinie zur Allergieprävention ( $\mathrm{Nr}$. 061/016). In Bezug auf die Diagnostik gibt es eine S2-Leitlinie zu Hauttests zur Diagnostik von allergischen Soforttyp-Reaktionen (Nr. 061-026) und eine gerade abgelaufene S1-Leitlinie zur In-vitro-Allergiediagnostik (Nr. 061/017). Zur spezifischen Therapie gibt es die S2-Leitlinie zur spezifischen Immuntherapie (Nr. 061-004). Eine multidisziplinäre S3-Leitlinie „Rhinosinusitis“ ist in Arbeit (Nr. 053-012). Von der Deutschen Gesellschaft für Hals-Nasen-Ohren-Heilkunde, Kopf- und Hals-Chirurgie gibt es die aktuelle S2k-Leitlinie „Rhinosinusitis“ (017-049).

\section{Begutachtung}

Die HNO-Begutachtung der allergischen RS findet ausreichend Hinweise in der Gutachtenliteratur. Feldmann verweist auf die Anhaltspunkte für die ärztliche Gutachtertätigkeit im sozialen Entschädigungsrecht und im Schwerbehindertenrecht. Hiernach kann eine Verengung der Nasengänge einseitig je nach Atembehinderung mit 0-10 (GdB/MdE-Grad), doppelseitig mit leichter bis mittelgradiger Atembehinderung mit 10, und doppelseitig mit starker Atembehinderung mit 20 bewertet werden. Eine CRS leichteren Grades (ohne wesentliche Neben- und Folgeerscheinungen) wird mit 0-10 und schweren Grades (ständige erhebliche Eiterabsonderung, Trigeminusreizerscheinungen, Polypenbildung) mit 20-40 bewertet. Ein assoziierter völliger Verlust des Riechvermögens mit der damit verbundenen Beeinträchtigung der Geschmackswahrnehmung kann mit bis zu 15 bewertet werden. Der Aspekt der allergischen Ätiologie kann für die Beurteilung der Dauerhaftigkeit der Gesundheitsstörung und in Bezug auf eine mögliche Besserung relevant sein.

\section{Perspektiven}

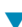

Für die Behandlung der allergischen Rhinitis wird primär die weitere Standardisierung der Be- handlung durch weitere Verbreitung der aktuellen Leitlinien wichtig sein [34]. Viel erhofft man sich von neuen medikamentösen Behandlungsmöglichkeiten [35]. Als Beispiel sei die AntiIgE-Therapie genannt, wobei die bisherigen Behandlungsergebnisse sehr variabel sind [36, 37]. Bei der CRS steht im Vordergrund, die Erkrankung und die Rolle der Schleimhautentzündung mit und ohne Nasenpolypen besser zu verstehen [38]. Möglicherweise wird es sinnvoll sein, die CRS nach neuen Endotypen zu klassifizieren, da die Patienten unterschiedlich auf verschiedene medikamentöse Behandlungsformen ansprechen. So wird bspw. vorgeschlagen, die CRS mit Nasenpolypen nach Expressionsprofilen der Nasenpolypen zu klassifizieren oder auch im Blut zirkulierende Biomarker für die Einteilung zu verwenden [39].

Ein wichtiges Thema bleibt die effektive Erreichbarkeit der Nasennebenhöhlen durch eine topische Therapie. Nasensprays mit liposomaler Aufbereitung der Medikamente könnten eine interessante Option werden, um eine bessere Wirkung direkt an der Nasennebenhöhlenschleimhaut zu erreichen [40]. Gerade für die postoperative Anwendung nach Nasennebenhöhlenchirurgie scheint die Anwendung von pulsierenden Aerosolen eine vielversprechende Perspektive zu sein [41].

\section{Literatur}

1 Bousquet J, Khaltaev N, Cruz AA et al. Allergic Rhinitis and its Impact on Asthma (ARIA) 2008 update (in collaboration with the World Health Organization, GA(2)LEN and AllerGen). Allergy 2008; 63: 8-160

2 Brozek JL, Bousquet J, Baena-Cagnani CE et al. Allergic Rhinitis and its Impact on Asthma (ARIA) guidelines: 2010 revision. J Allergy Clin Immunol 2010; 126: 466-476

3 Stuck BA, Bachert C, Federspil P et al. Rhinosinusitis guidelines - unabridged version: S2 guidelines from the German Society of Otorhinolaryngology, Head and Neck Surgery. HNO 2012; 60: 141-162

4 Riechelmann $\mathrm{H}$. Europäische Akademie fur Allergie und Klinische Immunologie und der European Rhinologic Society. Chronic Rhinosinusitis - EPOS 2012 Part I. Laryngorhinootologie 2013; 92: 193-201 quiz 202-203

5 Riechelmann H, Giotakis A, Kral F. Acute rhinosinusitis in adults - EPOS 2012 Part II. Laryngorhinootologie 2013; 92: 763-776

6 Fokkens WJ, Lund VJ, Mullol J et al. EPOS 2012: European position paper on rhinosinusitis and nasal polyps 2012. A summary for otorhinolaryngologists. Rhinology 2012; 50: 1-12

7 DeYoung K, Wentzel JL, Schlosser RJ et al. Systematic review of immunotherapy for chronic rhinosinusitis. Am J Rhinol Allergy 2014; 28: 145-150

8 Kim YH, Jang TY. Usefulness of the subjective cold hyperresponsiveness scale as evaluated by cold dry air provocation. Am J Rhinol Allergy 2012; 26: 45-48

9 Bousquet J, Heinzerling L, Bachert C et al. Practical guide to skin prick tests in allergy to aeroallergens. Allergy 2012; 67: 18-24

10 Stuck BA, Bachert C, Federspil P et al. Rhinosinusitis guidelines of the German Society for Otorhinolaryngology, Head and Neck Surgery. HNO 2007; 55: 758-760 762-764, 766-777 
11 Liu $Q$ Lu X, Bo $M$ et al. The microbiology of chronic rhinosinusitis with and without nasal polyps. Acta Otolaryngol 2014; 134: 1251-1258

12 Tan BK, Chandra RK, Pollak J et al. Incidence and associated premorbid diagnoses of patients with chronic rhinosinusitis. J Allergy Clin Immunol 2013; 131: $1350-1360$

13 Hur K, Liang J, Lin SY. The role of secondhand smoke in allergic rhinitis: a systematic review. Int Forum Allergy Rhinol 2014; 4: 110-116

14 Hur K, Liang J, Lin SY. The role of secondhand smoke in sinusitis: a systematic review. Int Forum Allergy Rhinol 2014; 4: 22-28

15 Sidell D, Shapiro NL, Bhattacharyya N. Obesity and the risk of chronic rhinosinusitis, allergic rhinitis, and acute otitis media in school-age children. Laryngoscope 2013; 123: 2360-2363

16 Terreehorst I, Hak E, Oosting AJ et al. Evaluation of impermeable covers for bedding in patients with allergic rhinitis. New Engl J Med 2003; 349: 237-246

17 Hermelingmeier KE, Weber RK, Hellmich $M$ et al. Nasal irrigation as an adjunctive treatment in allergic rhinitis: a systematic review and meta-analysis. Am J Rhinol Allergy 2012; 26: e119-e125

18 Schlenter WW, Blessing $R$, Heintz B. Conservative treatment of chronic sinusitis. Success of oral bacterial lysate therapy. Laryngorhinootologie 1989; 68 : 671-674

19 Kaplan A. Canadian guidelines for chronic rhinosinusitis: Clinical summary. Can Fam Physician 2013; 59: 1275-1281 e1528-e1234

20 Meadows A, Kaambwa B, Novielli $N$ et al. A systematic review and economic evaluation of subcutaneous and sublingual allergen immunotherapy in adults and children with seasonal allergic rhinitis. Health Technol Assess 2013; 17: vi, xi-xiv 1-322

21 Erekosima N, Suarez-Cuervo C, Ramanathan $M$ et al. Effectiveness of subcutaneous immunotherapy for allergic rhinoconjunctivitis and asthma: a systematic review. Laryngoscope 2014; 124: 616-627

22 Grundmann SA, Mosters P, Brehler R. Specific immunotherapy. Hautarzt 2014; 65: 633-645 quiz 646-647

23 Matricardi PM, Kuna P, Panetta V et al. Subcutaneous immunotherapy and pharmacotherapy in seasonal allergic rhinitis: a comparison based on meta-analyses. J Allergy Clin Immunol 2011; 128: 791-799 e796

24 Radulovic $S$, Wilson D, Calderon $M$ et al. Systematic reviews of sublingual immunotherapy (SLIT). Allergy 2011; 66: 740-752

25 Horak F, Zieglmayer P, Zieglmayer $R$ et al. Early onset of action of a 5-grass-pollen 300-IR sublingual immunotherapy tablet evaluated in an allergen challenge chamber. J Allergy Clin Immunol 2009; 124: 471-477 e471

26 Feng $S, X u Y, M a R$ et al. Cluster subcutaneous allergen specific immunotherapy for the treatment of allergic rhinitis: a systematic review and meta-analysis. PloS one 2014; 9: e86529

27 Jose J, Coatesworth AP. Inferior turbinate surgery for nasal obstruction in allergic rhinitis after failed medical treatment. The Cochrane database of systematic reviews 2010

28 Rudmik L, Mace J, Soler ZM et al. Long-term utility outcomes in patients undergoing endoscopic sinus surgery. Laryngoscope 2014; 124: 19-23

29 Rudmik L, Soler ZM, Mace JC et al. Economic evaluation of endoscopic sinus surgery versus continued medical therapy for refractory chronic rhinosinusitis. Laryngoscope 2015; 125: 25-32

30 Georgalas C, Cornet M, Adriaensen G et al. Evidencebased surgery for chronic rhinosinusitis with and without nasal polyps. Curr Allergy Asthma Rep 2014; 14: 427

31 Hellings PW, Fokkens WJ, Akdis C et al. Uncontrolled allergic rhinitis and chronic rhinosinusitis: where do we stand today? Allergy 2013; 68: 1-7
32 Veling MC. The role of allergy in pediatric rhinosinusitis. Curr Opin Otolaryngol Head Neck Surg 2013; 21: $271-276$

33 Stenner M, Rudack C. Diseases of the nose and paranasal sinuses in childhood. Laryngorhinootologie 2014; 93: 24-48

34 Braido F, Sclifo F, Ferrando $M$ et al. New therapies for allergic rhinitis. Curr Allergy Asthma Rep 2014; 14: 422

35 Braido F, Arcadipane F, Marugo F et al. Allergic rhinitis: current options and future perspectives. Curr Opin Allergy Clin Immunol 2014; 14: 168-176

36 Bonini M, Di Maria G, Paggiaro P et al. Potential benefit of omalizumab in respiratory diseases. Ann Allergy Asthma Immunol 2014; 113: 513-519

37 Solelhac $G$, Charpin D. Management of allergic rhinitis. F1000Prime Rep 2014; 6: 94

38 Bachert C, Van Bruaene N, Toskala E et al. Important research questions in allergy and related diseases: 3-chronic rhinosinusitis and nasal polyposis - a GALEN study. Allergy 2009; 64: 520-533

39 Akdis CA, Bachert C, Cingi $C$ et al. Endotypes and phenotypes of chronic rhinosinusitis: a PRACTALL document of the European Academy of Allergy and Clinical Immunology and the American Academy of Allergy, Asthma \& Immunology. J Allergy Clin Immunol 2013; 131: 1479-1490

40 Eitenmuller A, Piano L, Bohm M et al. Liposomal Nasal Spray versus Guideline-Recommended Steroid Nasal Spray in Patients with Chronic Rhinosinusitis: A Comparison of Tolerability and Quality of Life. J Allergy (Cairo). 2014; 2014:146280. DOI: 10.1155/ 2014/146280. Epub 2014 May 22

41 Moller W, Schuschnig U, Celik G et al. Topical drug delivery in chronic rhinosinusitis patients before and after sinus surgery using pulsating aerosols. PloS one 2013; 8: e74991

42 Bousquet J, Reid J, van Weel C et al. Allergic rhinitis management pocket reference 2008. Allergy 2008; 63: 990-996

43 Mafee MF, Valvassori GE, Becker M. Imaging of the Head and Neck. Stuttgart: Thieme, 2005

\section{Über die Autoren}

$\nabla$

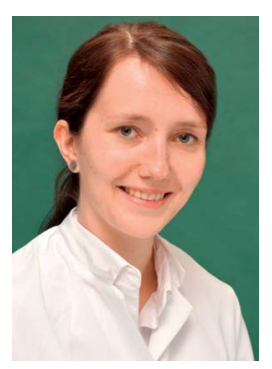

Katharina Geißler

Jahrgang 1985, Dr. med. 2004-2010 Medizinstudium an der Martin-Luther-Universität Halle-Wittenberg, dort 2012 Promotion über tumorinfiltrierende Lymphozyten bei Nierentumoren. Seit 2011 Assistenzärztin an der Klinik und Poliklinik für Hals-, Nasen-, Ohrenheilkunde des Universitätsklinikum Jena. Klinische Schwerpunkte: effektive Schmerzbehandlung nach Tonsillektomie, Allergologie, Schlafmedizin. Experimentelle Schwerpunkte: Entwicklung eines Entzündungsmodells hinsichtlich der T-Zellfunktion an humanen Gaumentonsillen. 


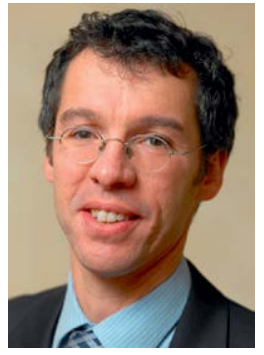

Orlando Guntinas-Lichius Jahrgang 1967, Univ.-Prof. Dr. med., Direktor der Klinik und Poliklinik für Hals-, Nasen-, Ohren-Heilkunde des Universitätsklinikums Jena. 1987-1993 Studium der Humanmedizin in Köln, 1994 Promotion über experimentelle Untersuchungen zur Hypoglossus-Facialis-Nervennaht. 1994-1995 Arzt im Praktikum in der Hals-Nasen-Ohren-Klinik der Universität zu Köln und anschließend dort Assistenzarzt. 1998 Anerkennung der Gebietsbezeichnung Hals-Nasen-Ohren-Heilkunde. 2001 Habilitation für das Fach Hals-Nasen-Ohren-Heilkunde, ebenfalls in Köln, und Ernennung zum Oberarzt. 2003 Leitender Oberarzt. 2006 Außerplanmäßige Professur der Universität zu Köln. Seit 2006 W3-Professur für HNO-Heilkunde an der Universität Jena und Direktor der dortigen HNO-Klinik. Seit 2009 Mitglied der Deutschen Akademie der Naturforscher Leopoldina und seit
2010 Prodekan für Lehre. Klinische Schwerpunkte: neue diagnostische Verfahren und klinische Prüfungen zur multimodalen Therapie von Kopf-Hals-Tumoren, Entwicklung neuer Verfahren zur Rekonstruktion des Nervus facialis, funktionelle Diagnostik und Therapie von multisensorischen Geschmacksstörungen und Multisensorik der Gleichgewichtsfunktion. Experimentelle Schwerpunkte: Verbesserung der peripheren Nervenregeneration, insbesondere des N. facialis.

\section{Korrespondenzadresse}

Univ.-Prof. Dr. med. Orlando GuntinasLichius

Klinik und Poliklinik für Hals-, Nasen-, Ohrenheilkunde

Universitätsklinikum Jena

Lessingstraße 2

D-07740 Jena

orlando.guntinas@med.uni-jena.de 


\section{CME-Fragen Allergische Rhinitis im Kontext der chronischen Rhinosinusitis}

Welche Aussage zur Rhinitis/Rhinosinusitis trifft nicht zu?

A Eine akute Rhinosinusitis bezeichnet eine Rhinosinusitis mit Dauer von weniger als 12 Wochen.

B Eine rezidivierende Rhinosinusitis bezeichnet eine akute Rhinosinusitis mit 2 Episoden im Jahr.

C Eine chronische Rhinosinusitis bezeichnet eine Rhinosinusitis mit Dauer von mind. 12 Wochen.

D Eine intermittierende allergische Rhinitis bezeichnet eine Rhinitis mit allergischen Symptomen von weniger als 4 Tagen/Woche oder weniger als 28 Tagen/Jahr.

E Eine persistierende allergische Rhinitis bezeichnet eine Rhinitis mit allergischen Symptomen von mind. 4 Tagen/Woche oder mindestens 28 Tagen/Jahr.

Welche Aussage zu den Allergieformen nach Coombs und Gell trifft nicht zu?

A Die Typ-I-Reaktion basiert auf einer IgE-vermittelten Sofortreaktion.

B Ein Beispiel für eine Typ-II-Reaktion ist ein Transfusionszwischenfall.

c Ein typisches Beispiel für eine Typ-III-Reaktion ist ein Kontaktekzem.

D Eine Typ-IV-Reaktion tritt typischerweise nach 24-72 Stunden auf.

E Eine Typ-II-Reaktion wird durch zytotoxische IgG- und IgM-Antikörper vermittelt.

3 Welche Aussage zu Epidemiologie und Ätiologie der allergischen Rhinitis nicht trifft zu?

A Die Entwicklung einer allergischen Rhinitis resultiert aus einem multikausalen Geschehen.

B Der Einfluss von aktivem und passivem Rauchen ist belegt.

C Die Prävalenz der allergischen Rhinitis liegt in Deutschland bei $5-10 \%$.

D Patienten mit chronischer Rhinosinusitis und Asthma bronchiale weisen $\mathrm{zu} \mathrm{30 \%} \mathrm{eine} \mathrm{Analgetika-Toleranz}$ auf.

E Zur Hygienehypothese gibt es teilweise widersprüchliche Untersuchungsergebnisse.

Welches ist kein Kardinalsymptom derallergischen Rhinitis?
A Hyposmie
B Niesen
C Juckreiz der Nase
D klare Sekretion
E nasale Obstruktion

5 Was ist kein typischer HNO-ärztlicher Befund der allergischen Rhinitis?

A Schwellung der unteren Nasenmuschel

B eitrige Sekretion

C Schwellung der mittleren Nasenmuschel

D ödematöse, bläulich-livide Schleimhaut

E ekzematöse Veränderung der Haut um den Naseneingang

6 Welche Aussage zu Testverfahren zur Diagnose einer allergischen Rhinitis trifft zu?

A Eine Hauttestung (z.B. Pricktest) selbst kann nicht zu einer anaphylaktischen Reaktion führen.

B Es ist unbedeutsam, welche Medikamente der Patient dauerhaft einnimmt.

c Ein Pricktest wird üblicherweise an der Palmarseite des Unterarms durchgeführt.

D Der am häufigsten durchgeführte Test zur Diagnose einer allergischen Rhinitis ist der Epikutantest.

E Die serologische Bestimmung des Gesamt-IgEs sowie spezifischer IgE-Antikörper kann die Diagnose einer allergischen Rhinitis unterstützen.

Welche Aussage trifft für den nasalen Provokationstest nicht zu?

A Der nasale Provokationstest kann hilfreich sein, wenn die Befundkonstellation zur Diagnose einer allergischen Rhinitis unklar oder widersprüchlich ist.

B Der nasale Provokationstest kann hilfreich sein, wenn die Patienten nach Hauttestung und/oder Labordiagnostik mehrere Allergene aufweisen und die klinisch relevanten Allergene erkannt werden sollen.

C Der nasale Provokationstest hat eine hohe Sensibilität und Spezifität.

D Der nasale Provokationstest sollte bei einer Raumtemperatur von $20 \pm 1,5{ }^{\circ} \mathrm{C}$ durchgeführt werden.

E Beim nasalen Provokationstest ist es nicht bedeutsam, welche Medikamente der Patient dauerhaft einnimmt.

8 Welche Aussage zu wichtigen Differenzialdiagnosen der allergischen Rhinitis und chronischen Rhinosinusitis trifft nicht zu?

A Eine ziliäre Dyskinesie oder zystische Fibrose kann Ursache einer chronischen Rhinosinusitis sein.

B Systemische Erkrankungen wie der M. Wegener, das Churg-Strauss-Syndrom oder eine Sarkoidose können Ursache einer chronischen Rhinosinusitis sein.

c Patienten mit einer Analgetika-Intoleranz zeigen Symptome wie nasale Obstruktion, Rhinorrhoe, Hyposmie, Polyposis nasi und Asthma bronchiale.

D Eine pilzbedingte chronische Rhinosinusitis ist in Deutschland eine häufige Erkrankung.

E Patienten mit Analgetika-Intoleranz können auch gastrointestinale Entzündungsreaktionen bis Ulzerationen, Angioödeme und Urtikaria aufweisen. 
9 Welche Aussage zur Prävention einer allergischen Rhinitis trifft zu?

A Maßnahmen zur Primär- und Sekundärprävention haben in Deutschland bisher keine hohe Wichtigkeit.

B Stillen in den ersten 4 Lebensmonaten ist nicht effektiv zur Prävention einer allergischen Rhinitis.

C Aktive und passive Exposition gegenüber Tabakrauch erhöht das Allergierisiko.

D Adipositas im Kindesalter ist ein Risikofaktor für die allergische Rhinitis.

E Eine Leitlinie zur Allergieprävention existiert bisher nicht.
10 Welche Aussage zur Therapie einer allergischen Rhinitis trifft zu?

A Eine Karenz des auslösenden Allergens kann keinen ausreichenden Effekt erzielen.

B Leiden Patienten auch unter Augensymptomen der allergischen Rhinitis, ist bisher kein Medikament bekannt, das die Symptome lindern kann.

C Eine spezifische Immuntherapie sollte nicht länger als 1 Jahr angewendet werden.

D Eine sublinguale Immuntherapie ist immer besser als eine subkutane Immuntherapie.

E Orale und topische H1-Antihistaminika können zur Symptomlinderung der allergischen Rhinitis beitragen. 


\section{CME-Fortbildung mit der Laryngo-Rhino-Otologie}

Zertifizierte Fortbildung Hinter der Abkürzung CME verbirgt sich „continuing medical education“, also kontinuierliche medizinische Fort- und Weiterbildung. Zur Dokumentation der kontinuierlichen Fortbildung der Ärzte wurde das Fortbildungszertifikat der Ärztekammern etabliert. Hauptzielgruppe für das Fortbildungszertifikat sind Ärzte mit abgeschlossener Facharztausbildung, die im 5-jährigen Turnus einen Fortbildungsnachweis erbringen müssen. Es ist jedoch auch für Ärzte in der Facharztweiterbildung gedacht.

Die Fortbildungseinheit In den einheitlichen Bewertungskriterien der Bundesärztekammer ist festgelegt: „Die Grundeinheit der Fortbildungsaktivitäten ist der Fortbildungspunkt. Dieser entspricht in der Regel einer abgeschlossenen Fortbildungsstunde (45 Minuten)“.

Für die erworbenen Fortbildungspunkte muss ein Nachweis erbracht werden. Hat man die erforderliche Anzahl von 250 Punkten gesammelt, kann man das Fortbildungszertifikat bei seiner Ärztekammer beantragen, welches man wiederum bei der KV (niedergelassene Ärzte) oder bei seinem Klinikträger (Klinikärzte) vorlegen muss.

Anerkennung der CME-Beiträge Die Fortbildung in der Laryngo-Rhino-Otologie wurde von der Nordrheinischen Akademie für Ärztliche Fort- und Weiterbildung für das Fortbildungszertifikat anerkannt, das heißt, die Vergabe der Punkte kann direkt durch die Thieme Verlagsgruppe erfolgen. Die Fortbildung in der Laryngo-Rhino-Otologiegehört zur Kategorie „strukturierte interaktive Fortbildung“. Entsprechend einer Absprache der Ärztekammern werden die von der Nordrheinischen Akademie für Ärztliche Fort- und Weiterbildung anerkannten Fortbildungs- veranstaltungen auch von den anderen zertifizierenden Ärztekammern anerkannt.

Datenschutz Ihre Daten werden ausschließlich für die Bearbeitung dieser Fortbildungseinheit verwendet. Es erfolgt keine Speicherung der Ergebnisse über die für die Bearbeitung der Fortbildungseinheit notwendige Zeit hinaus. Die Daten werden nach Versand der Testate anonymisiert. Namens- und Adressangaben dienen nur dem Versand der Testate. Die Angaben zur Person dienen nur statistischen Zwecken und werden von den Adressangaben getrennt und anonymisiert verarbeitet.

Teilnahme Jede Ärztin und jeder Arzt soll das Fortbildungszertifikat erlangen können. Deshalb ist die Teilnahme am CME-Programm der Laryngo-Rhino-Otologie nicht an ein Abonnement geknüpft! Die Teilnahme ist im Internet (http://cme.thieme.de) möglich.

Im Internet muss man sich registrieren, wobei die Teilnahme an Fortbildungen abonnierter Zeitschriften ohne Zusatzkosten möglich ist.

Teilnahmebedingungen Für eine Fortbildungseinheit erhalten Sie 3 Fortbildungspunkte im Rahmen des Fortbildungszertifikates. Hierfür müssen 70\% der Fragen richtig beantwortet sein.

CME-Wertmarke für Nicht-Abonnenten Teilnehmer, die nicht Abonnenten der Laryngo-Rhino-Otologie sind, können für die Internet-Teilnahme dort direkt ein Guthaben einrichten, von dem pro Teilnahme ein Unkostenbeitrag abgebucht wird.

Teilnahme online möglich unter http://cme.thieme.de 